\begin{tabular}{|l|l|}
\hline $\begin{array}{l}\text { Postprint } \\
\text { Version }\end{array}$ & 1.0 \\
\hline Journal website & http://www.sciencedirect.com/science/article/pii/S0005796713000338 \\
\hline Pubmed link & $\underline{\text { http://www.ncbi.nlm.nih.gov/pubmed/23639303 }}$ \\
\hline DOI & 10.1016/j.brat.2013.02.001
\end{tabular}

This is a NIVEL certified Post Print, more info at http://www.nivel.eu

\title{
The role of the therapeutic relationship in cognitive behaviour therapy for chronic fatigue syndrome
}

\author{
MARIANNE J. HeIns, HANS KNOOP, GIJS BleIJENBERG \\ Expert Centre for Chronic Fatigue, Radboud University Nijmegen Medical Centre, 4628, PO \\ Box 9101, 6500 HB Nijmegen, The Netherlands
}

\begin{abstract}
Cognitive behaviour therapy (CBT) for chronic fatigue syndrome (CFS) can reduce fatigue and impairment. Recently, it was found that changes in fatigueperpetuating factors, i.e. focusing on symptoms, control over fatigue, perceived activity and physical functioning, are associated with and explain up to half of the variance in fatigue during CBT for CFS. The therapy relationship, e.g. outcome expectations and working alliance, may also contribute to treatment outcome. We aimed to examine the role of the therapy relationship in CBT and determine whether it exerts its effect independently of changes in fatigueperpetuating factors. We used a cohort of 217 CFS patients in which the pattern of change in fatigue-perpetuating factors was examined previously. Fatigue, therapy relationship and fatigue-perpetuating factors were measured at the start of therapy, three times during CBT and at the end of therapy. Baseline outcome expectations and agreement about the content of therapy predicted post therapy fatigue. A large part of the variance in post-treatment fatigue (25\%) was jointly explained by outcome expectations, working alliance and changes in fatigueperpetuating factors. From this, we conclude that positive outcome expectations and task agreement seem to facilitate changes in fatigue-perpetuating factors during CBT for CFS. It is therefore important to establish a positive therapy relationship early in therapy.
\end{abstract}
Abbreviations
CAL, causal attribution list;
CBT, cognitive behaviour therapy;
CFS, chronic fatigue syndrome; 
Heins, M.J., Knoop, H., Bleijenberg, G. The role of the therapeutic relationship in cognitive behaviour therapy for chronic fatigue syndrome. Behaviour Research and Therapy: 2013, 51(7), ) 368-376

CIS,Checklist Individual Strength;

SF36, Medical Outcomes Survey Short Form 36;

SIP, sickness impact profile;

WAI-s, Working Alliance Inventory - Short Form

\section{INTRODUCTION}

Chronic fatigue syndrome (CFS) is characterised by severe and medically unexplained fatigue that lasts longer than 6 months and results in severe limitations in daily life (Fukuda et al., 1994; Reeves et al., 2003). Cognitive behaviour therapy (CBT) for CFS can effectively reduce fatigue and impairment (Castell, Kazantzis, \& Moss-Morris, 2011; Chambers, Bagnall, Hempel, \& Forbes, 2006; Price, Mitchell, Tidy, \& Hunot, 2008). Even full recovery, defined as no longer being severely fatigued, being able to resume all activities, and having a perception of health and fatigue that is similar to healthy persons, is possible for a subgroup of patients (Knoop, Bleijenberg, Gielissen, van der Meer, \& White, 2007). Several models underlying CBT for CFS assume that there are certain fatigue-related beliefs and behaviour that perpetuate symptoms (Fry \& Martin, 1996; Gaab, 2004; Surawy, Hackmann, Hawton, \& Sharpe, 1995; Vercoulen et al., 1998; Wessely, David, Butler, \& Chalder, 1989). One of these models is that of Vercoulen et al. (1998). It is the only model that was statistically tested and it states that a high level of somatic attributions and focusing on symptoms and a low level of physical activity and low perceived control over symptoms lead to perpetuation of fatigue.

Recently, Wiborg, Knoop, Prins \& Bleijenberg (2011); Wiborg, Knoop, Frank \& Bleijenberg (2012); Wiborg, Knoop, stulemeijer et al. (2010) used mediation analyses of previous randomised controlled trials (RCTs) to test whether 1) CBT indeed leads to a change in these fatigue-perpetuating factors and 2) whether this change was related to the reduction in fatigue. Their studies showed that changes in focusing on symptoms and sense of control over fatigue indeed mediate the change in fatigue during CBT for CFS (Wiborg, Knoop, et al., 2012; Wiborg, et al. 2011). However, it is not the actual change in physical activity, but the perceived increase in activity that mediates the decrease in fatigue (Wiborg, Knoop, et al., 2012; Wiborg, Knoop, Stulemeijer, Prins, \& Bleijenberg, 2010). Heins et al. furthered their work by studying how these fatigue-perpetuating factors change during CBT for CFS. Their study showed that these variables change in the same period in which fatigue decreases. Changes in fatigue-perpetuating factors explained up to half of the variance in fatigue during CBT for CFS (Heins et al. submitted for publication). So although these studies provided insight into the changes that lead to a decrease in fatigue, these changes could only partly explain the treatment outcome of CBT for CFS.

Besides specific treatment techniques, extant research suggests that the therapy relationship, i.e. "the feelings and attitudes that therapist and client have toward one another and how these are expressed" (Norcross, 2001), is also an important contributor to treatment outcome in a variety of psychotherapies. A recent task force on evidence-based therapy relationships even concluded that "the therapy relationship accounts for why clients improve (or fail to improve) at least as much as 
Heins, M.J., Knoop, H., Bleijenberg, G. The role of the therapeutic relationship in cognitive behaviour therapy for chronic fatigue syndrome. Behaviour Research and Therapy: 2013, 51(7), 368-376

the particular treatment method" (Norcross \& Wampold, 2011). Wampold and Budge recently proposed a model of the therapy relationship, which consists of the bond between patient and therapist, setting of goals and tasks and expectations with respect to treatment outcome (Wampold \& Budge, 2012). This model thus includes outcome expectations and three elements of the working alliance as proposed by Bordin: task, goal and bond (Bordin, 1979). Tasks are what therapist and patient agree that needs to be done to reach the patient's goals. Goals are what the patient hopes to gain from therapy. Bond is the trust and confidence in the therapist. Although both outcome expectations and working alliance are considered empirically supported relational attributes across studies of various therapies (Constantino, Arnkoff, Glass, Ametrano, \& Smith, 2011;Fluckiger, Del Re, Wampold, Symonds, \& Horvath, 2012; Horvath, Del Re, Fluckiger, \& Symonds, 2011), their application is developed and maintained in different ways within different therapeutic models and protocols (Hatcher \& Barends, 2006; Ulvenes et al., 2012; Wampold \& Budge,

2012). We'll therefore briefly discuss the application of outcome expectations and working alliance in CBT in general and CBT for CFS specifically. In the protocol used for this study, recovery is considered the highest achievable outcome of CBT. Patients, however, may be hesitant to believe that full recovery is possible and may only expect to learn to cope with their chronic fatigue. Both the aetiology of CFS and its treatment are hotly debated (Action for ME/Association of Young People with ME, 2008; Kean, 2010; Working group on CFS/ME (Chronic Fatigue Syndrome/Myalgic Encephalomyelitis), 2002). Many CFS patients attribute their symptoms to a physical cause, such as a viral infection or immunological disorder (Deale, Chalder, \& Wessely, 1998). Patients with strong somatic attributions will, understandably, not immediately have faith in a cognitive behavioural therapist and the rationale of CBT. Indeed, the placebo response, which is strongly related to outcome expectations (Di Blasi, Harkness, Ernst, Georgiou, \& Kleijnen, 2001), is lower in psychological interventions for CFS than in somatic interventions (Cho, Hotopf, \& Wessely, 2005). Furthermore, in a recent large RCT only 57\% of the CFS patients allocated to CBT indicated that they were confident about the treatment (White et al., 2011). The fact that patients are often referred for CBT after they have had symptoms for several years (see for example the study of Prins et al. (2001) with a median symptom duration of 4.9 years) may further lower outcome expectations. The working alliance in CBT is mainly reflected in collaborative empiricism, i.e. patient and therapist collaborating to uncover negative automatic thoughts through Socratic reasoning and homework assignments (Dattilio \& Hanna, 2012). Although in the theory underlying CBT it is assumed that a good working alliance in itself is insufficient to bring about change (Clark, Beck, \& Alford, 1999), a good alliance is considered necessary and has been related to positive treatment outcome (Horvath et al., 2011). The working alliance in CBT for CFS may not always be good as some CFS patients will have a different view than the CBT therapist about the cause of CFS (e.g. a somatic versus a cognitive behavioural model of CFS) and the goal of treatment (e.g. recovery versus coping with a chronic illness). The first objective of this paper is to determine whether outcome expectations and working alliance contribute to the reduction in fatigue during CBT for CFS. A second objective is to determine whether they have a direct effect on treatment outcome, independently of 
Heins, M.J., Knoop, H., Bleijenberg, G. The role of the therapeutic relationship in cognitive behaviour therapy for chronic fatigue syndrome. Behaviour Research and Therapy: 2013, 51(7), 368-376

changes in fatigue-perpetuating factors or exert their effect (also) indirectly through changes in fatigue-perpetuating factors.

\section{METHODS}

\section{Patients}

We assessed the effect of the therapeutic relationship in the same cohort of patients in which the pattern of change in fatigue-perpetuating factors was previously examined (Heins et al., submitted for publication). In this study, consecutively referred patients who started CBT at our tertiary CFS specialist care centre between April 2008 and September 2010 were eligible to participate if they:

- Met the criteria for CFS as stated by the US Centers for Disease Control and Prevention. These include the experience of severe and medically unexplained fatigue for at least six months that brings about severe impairment in daily living and is accompanied by four out of eight specific additional symptoms (Fukuda et al., 1994; Reeves et al., 2003). These are by far most often used in studies on CFS (Christley, Duffy, \& Martin, 2012).

Severe fatigue was defined as a score of $\geq 35$ on the Checklist Individual Strength (CIS), subscale fatigue severity (Vercoulen et al., 1994), and severe functional impairment was defined as a total score $\geq 700$ on the Sickness Impact Profile (SIP) (Bergner, Bobbitt, Pollard, Martin, \& Gilson, 1976; Jacobs, Luttik, Touw-Otten, \& de Melker, 1990; Knoop et al., 2007;Pollard, Bobbitt, Bergner, Martin, \& Gilson, 1976). If patients had not been extensively medically examined prior to referral, they first received a standard medical examination at the outpatient clinic of our department of internal medicine, to rule out any somatic disorder that excludes the diagnosis of CFS.

- Were at least 18 years old. There is a separate treatment protocol for adolescent patients at our centre.

- Were able to read and write Dutch.

Patients were excluded if they were currently engaged in a legal procedure for a disability claim until this procedure was completed.

The study was conducted according to the Helsinki declaration as revised in 1994, and approved by the ethical committee of the Radboud University Nijmegen Medical Centre. All participants gave written informed consent before participation.

\section{CBT}

The protocol of CBT for CFS used in this study (Bleijenberg et al., 2003; Knoop \& Bleijenberg, 2010) consists of approximately 12 sessions during 6 months. First of all, illness attributions are discussed with the patient, bearing in mind that a somatic cause for the symptoms has not been found in previous medical examination. The way through which thoughts and behaviour can lead to the persistence of symptoms is explained and the patient formulates his or her goals of therapy. These goals usually include the resumption of work, hobbies and other activities that imply recovery for the patient. Recovery is named as the maximal attainable goal of therapy, although patients are told that not every patient will reach this goal. After 
the formulation of treatment goals, bedtimes are regulated to stop disruption of the circadian rhythm. Next, patients are taught how to recognise and challenge nonaccepting and catastrophising thoughts in response to fatigue that may hamper adequate coping. They also learn to redirect their attention from the fatigue to activities and their environment. Patients who are relatively physically active (i.e. bursts of activity followed by inactivity) first start dividing their activity evenly throughout the day. Patients who have a low level of physical activity start immediately with the next part of the treatment, in which patients gradually increase their level of physical activity, mostly by walking or cycling. Dysfunctional illness beliefs, such as the thought that even moderate levels of activity are harmful, are challenged during this period. Once patients have increased their level of physical activity, they start to realise their treatment goals in the same way. At the end of treatment they are encouraged to stop labelling themselves as a CFS patient. All participating therapists were thoroughly trained in the protocol of CBT for CFS and received frequent intervision and supervision to ensure fidelity to the treatment protocol.

\section{Measurements}

Baseline and post-treatment fatigue were measured as a part of the standard diagnostic procedure at our centre. Baseline expectations and working alliance were assessed after the first therapy session, to ascertain that a baseline working alliance could have been established. As changes in outcome expectations and alliance after the first session may also affect treatment outcome, we conducted three interim measurements of fatigue, expectations and working alliance: 6, 12 and 18 weeks after the start of CBT. Patients received the questionnaires from the researcher and were told that their answers would not be communicated with their therapist. This was also mentioned in the written information about the study and in the written instructions given together with the questionnaires. Patients were informed that the study aimed to investigate how CBT for CFS works.

\section{Fatigue}

Fatigue was assessed with the subscale fatigue severity of the Checklist Individual Strength (CIS). This subscale indicates the level of fatigue in the previous two weeks, measured with eight items on a seven-point scale (range 8-56). A score of 35 and higher (i.e. 2 standard deviations above the mean of a healthy control group) indicates severe fatigue. Cronbach's alpha ranges from 0.83 to 0.92 (Vercoulen, Alberts, \& Bleijenberg, 1999; Vercoulen et al., 1994).

\section{Baseline characteristics}

\section{Somatic attributions}

Somatic attributions regarding the CFS symptoms were measured with the Causal Attributions List (CAL), which consists of five questions measured on a five-point Likert scale (range 5-20). Higher scores indicate more somatic attribution (Prins et al., 2001).

\section{Limitations in daily functioning}

Limitations in daily functioning were measured with the Sickness Impact Profile (SIP), (Bergner et al., 1976) which is a widely used multi-facetted questionnaire with 
Heins, M.J., Knoop, H., Bleijenberg, G. The role of the therapeutic relationship in cognitive behaviour therapy for chronic fatigue syndrome. Behaviour Research and Therapy: 2013, 51(7), 368-376

good reliability (Pollard et al., 1976). It measures limitations on different domains of daily living, such as sleep and rest, work, home management, mobility and social interaction. It has been repeatedly used in studies testing the efficacy of interventions for CFS. A score above 700 indicates severe limitations (van der Werf, de Vree, Alberts, van der Meer, \& Bleijenberg, 2002).

\section{Therapy relationship}

\section{Outcome expectations}

Patients' expectations about the outcome of therapy were measured with the Treatment Outcome Expectations Questionnaire, which was developed for this study. The original questionnaire consisted of 5 questions regarding expectations with respect to the outcome of the treatment: 1) Treatment will help me to get rid of my symptoms; 2) After treatment I will be less fatigued; 3) I'll learn to cope with my fatigue; 4) I'll learn to accept my fatigue; 5) After treatment I'll be recovered. These questions were answered on a four point Likert-scale ranging from 'I do not expect that at all' (0) to 'I most certainly expect that' (4). Higher scores indicate more positive outcome expectations. Therapists' expectations about the outcome of therapy were measured with the following question: 'Do you think that [name of patient] will be recovered after therapy?' Therapists answered this question on a $100 \mathrm{~mm}$ Visual Analogue Scale ranging from 'certainly not' (0) to 'most certainly' (100).

\section{Working alliance}

Working alliance was measured with the Working Alliance Inventory - Short Version (WAI-S) (Tracey \& Kokotovic, 1989). This questionnaire consists of a separate client and a therapist version and measures working alliance using 12 questions on a 5-point Likert scale ranging from 'never' to 'always'. Three subscales can be calculated; bond, goal agreement and task agreement. These subscales reflect the emotional bond with the therapist and the agreement about the goal of therapy and the tasks that have to be performed to reach this goal. Factor analysis showed that these three factors were also valid for the Dutch translation (Stinckens, Ulburghs, \& Claes, 2009). These factors are also relevant to our study. The task scale is relevant as patient and therapist may disagree about the content of the therapy, i.e. patients may believe that changing their thoughts and behaviour will not change their fatigue. The goal scale is relevant as patient and therapist may disagree about the goal of therapy, i.e. recovery vs. coping with chronic fatigue. Also the bond scale is useful. As we hypothesized in the introduction, the bond between patient and therapist may be less important in CBT for CFS as patients may see the therapist as someone explaining the treatment, however, this still has to be confirmed. Internal consistency of these factors was 0.85 for the task scale, 0.83 for the goal scale and 0.82 for the bond scale (Stinckens et al., 2009). In our study, this was 0.76, 0.73 and 0.75 respectively.

\section{Fatigue-perpetuating factors}


Heins, M.J., Knoop, H., Bleijenberg, G. The role of the therapeutic relationship in cognitive behaviour therapy for chronic fatigue syndrome. Behaviour Research and Therapy: 2013, 51(7), ) 368-376

Fatigue perpetuating factors were measured with the same instruments as in the previous study of Heins et al. (submitted for publication). Control over fatigue was measured with the self-efficacy scale (De Vree et al., 2002; Prins et al., 2001)

(Cronbachs alpha 0.68-0.77). Focusing on symptoms was measured with the subscale 'focusing on symptoms' of the Illness Management Questionnaire (Ray, Weir, Stewart, Miller, \& Hyde, 1993) (Cronbach's alpha 0.88). Self-perceived physical activity was measured with the Checklist Individual Strength subscale physical activity (Vercoulen et al., 1994). (Cronbach's alpha 0.81) Self-reported physical functioning were measured with the corresponding subscale of the Medical Outcomes Survey SF36 (Steward, Hays, Ware 1998) (Cronbach's alpha 0.91).

\section{Statistical analyses}

To determine the psychometric properties of the Treatment Outcome Expectations Questionnaire, a list specifically developed for the current study, we performed an exploratory varimax rotated factor analysis and determined internal consistency of the obtained factors.

To test whether baseline expectations and working alliance predicted treatment outcome we built a multilevel linear regression model. Therapist was defined as the second level, and patient as the first level. For these analyses, we selected all patients who had completed the post-treatment measurement. We used a stepwise approach in which we started with a null-model with post-treatment fatigue as dependent variable and the baseline characteristics fatigue, symptom duration, somatic attributions and physical functioning as independent variables. These baseline characteristics were hypothesized to either predict treatment outcome (baseline fatigue and physical functioning) or to be related to the therapy relationship (symptom duration and somatic attributions). In the second step we then added outcome expectations and working alliance of the patient, in the third step we added outcome expectations and working alliance as rated by the therapist. Using an F-test, we could statistically test the incremental proportions of variance in post-treatment fatigue attributable to each set of variables.

As changes during therapy may also affect the outcome of CBT, we also tested whether changes in outcome expectations and working alliance predicted posttreatment fatigue. We built separate multilevel linear regression models for each period (e.g. baseline - first interim measurement) in a stepwise approach similarly to that described for the pre-treatment variables in the previous paragraph. We first entered the significant baseline predictors of post-treatment fatigue and values of outcome expectations and working alliance at the start of the period, as a second step we entered the change in outcome expectations and working alliance during the period as rated by the patients and as a third step as rated by the therapists. Finally, we assessed the amount of variance in post-treatment fatigue explained by the therapy relationship and fatigue-perpetuating factors. As a first step we built a model with only those variables that significantly predicted post-treatment fatigue. We first entered baseline characteristics, then outcome expectations and working alliance at baseline, changes in outcome expectations and working alliance were entered as a separate step for each period, and finally we added the total change during therapy in fatigue-perpetuating factors that had previously been found to mediate the treatment effect of CBT for CFS (control over fatigue, focusing on 
symptoms, self-perceived physical activity and physical functioning). The proportion of variance in post-treatment fatigue each step explained independently was tested with an $F$-test comparing the full model to one without the step.

Descriptive statistical analyses were performed using SPSS 16.0 for Windows ${ }^{\circledR}$ and multi-level models were built using MLWin $2.24^{\circledR}$. All models were checked for multicollinearity (Tolerance $<0.10$ ). To correct for multiple testing we adjusted $p$ values using the False Discovery Rate method per model (i.e. the 3 baseline models, the models for change in period 1 , the models for change in period 2 and the final model) (Benjamini, 1995). An adjusted p-value below 0.05 was considered statistically significant.

\section{RESULTS}

\section{Patients}

Between April 2008 and December 2010, 297 patients with a diagnosis of CFS and meeting all other inclusion criteria started CBT at our tertiary CFS specialist care centre. Ten patients dropped out from therapy before informed consent was asked and fifty-five refused to participate. The reason that was given most often for refusal was the perceived effort of having to do extra assessments during treatment. Of the 232 patients who gave informed consent, 217 completed the post treatment measurement (and 183 also completed at least two measurements during the study). A flowchart of the selection process is given in Fig. 1 and demographic characteristics of the study participants are given in Table 1 . In total, 12 therapists participated in this study and they treated on average 18 patients (range 4-36).

\section{[FIGURE 1]}

\section{Factor analysis and internal consistency of Treatment Outcome Expectations Questionnaire}

We performed an exploratory factor analysis with varimax rotation on the Treatment Outcome Expectations Questionnaire. This analysis indicated a two-factor structure.

The first factor, named 'outcome expectations recovery', consisted of three questions: Treatment will help me to get rid of my symptoms; After treatment I will be less fatigued; After treatment I'll be recovered. Eigen-values were 0.87, 0.84 and 0.83 respectively and Cronbach's alpha was 0.83 . The second factor, named 'outcome expectations coping', consisted of the questions: I'll learn to cope with my fatigue; I'll learn to accept my fatigue. Factor loadings were 0.67 and 0.87 respectively and Cronbach's alpha was 0.55 . As we deemed this too low we used only the first factor for our analyses. Mean pre-treatment values are given in Table 1.

\section{Predictive value of the baseline therapy relationship for post-treatment fatigue} For these analyses we selected the patients who had completed the post treatment measurement $(n=217)$. In 20 patients some of the items or subscales of the assessment were not filled in. These values were replaced with the mean of the remaining items of the subscale or questionnaire. Baseline outcome expectations and working alliance are given in Table 1 . Values indicate that outcome expectations and 
working alliance were generally positive, as they lay in the upper half of the range of the questionnaires. Expectations of the therapists were extremely positive, as they expected a mean chance on recovery of $85 \%$, which is considerably more than the actual percentage of patients that were no longer severely fatigued and impaired after therapy (68\%, defined as a CIS fatigue $<35$ and Sip $<700$ ).

The null model showed that there was no variance at the therapist level (residual variance 0.00 , s.e. 0.00 ), so we decided to proceed with single level models. Regression analyses (see Table 2) show that baseline patient characteristics explained $8 \%$ of variance in post-treatment fatigue $(F=3.0, p=0.03)$ and therapy relationship as rated by the patient added $25 \%$ of explained variance $(F=19.1, p=0.001)$. Expectations of recovery and task agreement proved a significant predictor of post treatment fatigue. Therapist-rated variables only added $3.5 \%$ of explained variance; the $F$-test indicated that their addition did not improve the model significantly $(F=2.8, p=0.09)$.

\section{[TABLE 2]}

\section{Predictive value of changes in the therapy relationship for post-treatment fatigue}

For the longitudinal analyses, we selected the 183 patients who had at least two interim measurements. We did not use the third interim measurement, as 67 patients did not complete the third interim measurement because the post-therapy measurement had already been planned or they dropped out. Changes in the first period (baseline to interim measurement 1 ) in patient rated therapy relationship, primarily outcome expectations, added $6 \%$ of explained variance ( $F$ change $=4.6 ; p 0 .=0.004)$ to the reduction of fatigue. Adding therapist ratings only led to an additional 3\% of explained variance, which was not significant (Fchange 1.2; $p=0.39$ ). In the second period (interim measurement 1-2) similar results were seen. Changes in patient rated therapy relationship, again primarily outcome expectations, added $10 \%$ of explained variance in the reduction of fatigue ( $F$ change $=6.4 ; p=0.001$ ), but adding therapist ratings only led to an additional $2 \%$ of explained variance, which was not statistically significant ( $F$ change $1.3 ; p=0.35$ ) (Table 3a and Table 3b).

\section{[TABLE 3A][TABLE 3B]}

\section{The relative contribution of the therapy relationship and changes in fatigue perpetuating factors}

To assess the amount of variance in post-treatment fatigue explained by the therapy relationship and changes in fatigue-perpetuating factors, we built a model in which all factors that significantly predicted post-treatment fatigue in the previous models were added block-wise (i.e. first baseline characteristics, then therapy relationship at baseline, then changes in therapy relationship). The changes in fatigue-perpetuating factors were entered as a last step. This model explained $65 \%$ of post-treatment fatigue. A considerable proportion of variance was jointly explained by the therapy relationship and fatigue perpetuating factors (19\%). The independent contribution of the therapy relationship at baseline was only $4 \%(F=5.62 ; p=0.002)$, mostly attributable to therapist outcome expectations. Changes in the therapy relationship 
during therapy did not explain any of the variance independently $(F=0.33 p=0.67$ for the first period and $F=0.13 p=0.72$ for the second). Changes in fatigue perpetuating factors independently explained $32 \%$ variance in post-treatment fatigue $(F=38.8, p<0.001)$ (Table 4).

\section{[TABLE 4]}

\section{Discussion}

We studied, for the first time, the contribution of the therapy relationship to the reduction in fatigue during CBT for CFS in a large sample of patients and their therapists. Our study shows that especially patients' outcome expectations and task agreement at the start of treatment have a considerable influence on treatment outcome. They seem to exert their positive effect through a change in fatigue perpetuating factors.

\section{Contribution of therapy relationship to the reduction in fatigue during CBT for CFS}

Outcome expectations and working alliance, especially patients' outcome expectations and agreement about what they should do in therapy, already explain $25 \%$ of the variance in post-treatment fatigue at the beginning of therapy. This is not due to a lower level of complaints, more somatic attributions or longer disease duration in patients with lower outcome expectations. Further changes in outcome expectations have a modest additional effect on the decrease in fatigue.

Contrary to our expectations, most patients with CFS have positive outcome expectations when starting CBT. Therapists generally expect a very positive outcome of CBT. White et al. found considerably lower outcome expectations of patients in their randomised controlled trial on CBT for CFS than in the present study (White et al., 2011). This may be because patients who participated in their trial could not choose which treatment they would start. Participants in our trial all accepted a referral for CBT and among those referred, they may have been those most motivated and confident about CBT.

From the three domains of working alliance that we measured, only task agreement predicted treatment outcome. This factor reflects the amount of agreement between patient and therapist about the content of therapy, i.e. what the patient has to do to achieve his or her goals. This factor is most likely related to the perceived credibility of the rationale of CBT for CFS. Only if patients support the cognitive behavioural model of CFS will they believe that changing cognitions and behaviour will help to reduce their fatigue. Goal agreement is an important element of our protocol and the patient is encouraged to define concrete therapy goals, often including getting back to work or hobbies, together with the therapist. Goal agreement was not related to therapy outcome, so it does not seem to be agreement about the goals of therapy per se, but rather agreement about how to reach these goals that predicts a good outcome. Patients may also have agreed about therapy goals while not expecting to reach them. The bond with the therapist did not predict treatment outcome. This may be due to the nature of the symptoms. Patients with somatic symptoms may have a more mechanical vision on therapy than patients with e.g. depression or anxiety. They may feel that the therapist is merely someone explaining the treatment and that the bond with the therapist therefore is less important. 
Godfrey et al. (2007) studied the relationship between working alliance and treatment outcome in CBT for chronically fatigued patients of whom only a subset fulfilled criteria for CFS. They assessed roughly the same aspects of the working alliance as in the present study and named it working strategy consensus. This variable was also not related to treatment outcome. Other studies found a much larger effect of working alliance on treatment outcome of different psychotherapies (Gaston, Thompson, Gallagher, Cournoyer, \& Gagnon, 1998; Horvath \& Symonds, 1991). This may be related to the specific application of working alliance in CBT, in which collaborative empiricism has a large role (Dattilio \& Hanna, 2012). Only two questions of the WAI ask about collaboration, so this concept may not be covered by this questionnaire (Tee \& Kazantzis, 2011).

\section{Relative contribution of therapy relationship and changes in fatigue- perpetuating factors}

Most of the positive effect of outcome expectations and working alliance on fatigue is shared with that of changes in fatigue perpetuating factors, so both seem to be part of the same process. A positive therapy relationship thus certainly seems to be beneficial, but not sufficient to decrease fatigue. Some concurrent change in fatigue perpetuating factors is also necessary. The other way around is also true: a change in fatigue perpetuating factors alone only partly explains the change in fatigue. This implies that outcome expectations and alliance play a crucial role in facilitating cognitive change.

A recent study on CBT for anxiety indeed indicates that a positive therapy relationship facilitates cognitive changes (Hoffart, Borge, Sexton, Clark, \& Wampold, 2011), which then lead to a reduction in fatigue. A possible explanation for this finding could be that patients who have positive outcome expectations and who agree about the content of therapy are more engaged in therapy and are more compliant (Westra, Dozois, \& Marcus, 2007). Alternatively, patients with positive outcome expectations may be more likely to expect that they can learn to control their fatigue, which is one of the fatigue perpetuating factors in CBT for CFS.

\section{Limitations}

We assessed outcome expectations after the first session. Expectations may have already been influenced by the interventions of the therapist during the first session, e.g. by suggesting that recovery from CFS is possible. This may also explain the modest effect of changes during therapy. The advantage of measuring expectations after the first treatment session is that all patients have been properly informed and can base their expectations on reliable information. It would, however, be interesting to measure outcome expectations before and after the first session to determine changes in expectations brought on by the interaction with the therapist.

Outcome expectations were measured with questionnaires that have not previously

been validated. The subscale 'recovery' of the Treatment Outcome Expectations

Questionnaire showed good reliability. Therapists' outcome expectations were measured using a single Visual Analogue Scale, so reliability of this measurement may be low. Nevertheless, therapists' expectations of recovery were an important predictor of treatment outcome, independently of patients' expectations. We did not examine the predictive value of working alliance as seen by an independent assessor. Horvath et al. found that alliance best predicted treatment outcome when rated by the patient and least when rated by an independent observer (Horvath \& Symonds, 
Heins, M.J., Knoop, H., Bleijenberg, G. The role of the therapeutic relationship in cognitive behaviour therapy for chronic fatigue syndrome. Behaviour Research and Therapy: 2013, 51(7), ) 368-376

1991). Martin, Garske, \& Davis (2000) in a meta-analytic review, found however that the type of assessor (patient/therapist/independent) did not influence the relation between alliance and treatment outcome. This makes it unlikely that results from an independent assessor would have been entirely different.

Our study did not include a control group, however the fact that positive expectations at the start of the treatment are related to post treatment fatigue suggests a causal relationship. The fact that expectations no longer predicted post treatment fatigue when changes in fatigue-perpetuating factors were taken into account gives further support to the theory that positive expectations lead to a better treatment outcome through therapeutic processes and not because of other mechanisms unrelated to CBT.

Another limitation of our study is that patients who were less motivated for CBT and had less positive outcome expectations and working alliance may not have participated in our study. This may have led to a selection bias and may limit generalisability of our study results. However, assuming that we had a restricted range in outcome expectations and working alliance due to a selection bias makes it even more remarkable that we found an effect of outcome expectations in our sample. We did not measure patient participation within the session, but we measured patients' homework compliance. At the first measurement, $10 \%$ of the patients and $15 \%$ of the therapists stated that patients had never or only seldom done their homework, which increased to $11 \%$ and $25 \%$, respectively, at the second interim measurement. This indicates that patients were relatively adherent. However, numbers were too low to compare treatment outcome between those adherent and non-adherent to therapy.

It is possible that working alliance was higher for those patients working with therapists who more competently used thought evaluation techniques. We did not directly measure therapists' competence or adherence to the protocol, but the multilevel analysis indicated almost no variation in treatment outcome between therapists. The same was found by Cella, Stahl, Reme, \& Chalder, (2011). Wiborg, Knoop, Wensing, et al. (2012) did find differences in outcome between therapists. Their study was conducted in three secondary care centres in which CBT for CFS was being implemented. The centre in which therapists worked explained a part of the therapist effect. Both our study and that of Cella et al. were conducted in one highly specialised tertiary care centre, and all therapists participating in our study had been thoroughly trained in the protocol of CBT for CFS and received frequent intervision and supervision. This will probably have limited the differences between therapists and the way they performed CBT.

\section{Implications for clinical practice and future research}

As the present study indicates that outcome expectations at the start of therapy may facilitate positive changes in fatigue-perpetuating factors, interventions aimed at establishing positive outcome expectations should be given as early in the intervention as possible to optimise their effect. Greenberg et al. suggested some approaches to optimise patients expectations that can also be applied to CBT for CFS (Greenberg, Constantino, \& Bruce, 2006), such as installing a sense of hope, e.g. by changing illness representations of CFS that imply that someone has a chronic illness that is unchangeable into an illness that can be positively influenced by the patient. Extensively explaining the underlying rationale of CBT and explicitly asking for the 
Heins, M.J., Knoop, H., Bleijenberg, G. The role of the therapeutic relationship in cognitive behaviour therapy for chronic fatigue syndrome. Behaviour Research and Therapy: 2013, 51(7), 368-376

opinion of the patient and significant others to the rationale may help to improve outcome expectations.

Although some authors suggest otherwise (Van Houdenhove, 2006), our results that expectations of recovery are associated with therapy outcome suggest it is important that therapists discuss the possibility of a full recovery (Knoop et al., 2007). They can make the concept of recovery more tangible by letting the patient define what he or she would like to do when he/she did not have CFS anymore and translate this into concrete therapy goals.

\section{CONCLUSIONS}

Expectations of recovery and agreement about the content of treatment contribute to actual treatment outcome. This study enhances the understanding of the process of change during CBT for CFS as it shows that these factors do not have a direct positive effect on post-treatment fatigue, but seem to facilitate cognitive changes during CBT for CFS.

\section{FUNDING}

This study was funded by the Netherlands organisation for health research and development (ZON-MW). They had no role in study design, in the collection, analysis and interpretation of data, in the writing of the report or in the decision to submit the article for publication.

\section{CONFLICT OF INTEREST}

No interests to declare.

\section{ACKNOWLEDGEMENTS}

We would like to thank the participating patients and therapists for filling in all those questionnaires, Alastair Leyland and Peter Groenewegen for their assistance with the multi-level analyses, and special thanks to Judith de Natris, Lianne Vermeeren, Tiny Fasotti and Carel Kruip for their invaluable help in collecting the data.

\section{APPENDiX A. SUPPLEMENTARY DATA}

\section{REFERENCES}

Action for ME/Association of Young People with ME ME 2008: What Progress? (2008)

Y. Benjamini Controlling the false discovery rate: a practical and powerful approach to multiple testing Journal of the Royal Statistical Society, 57 (1995), pp. 289-300

M. Bergner, R.A. Bobbitt, W.E. Pollard, D.P. Martin, B.S. Gilson The sickness impact profile: validation of a health status measure Medical Care, 14 (1976), pp. 57-67

G. Bleijenberg, J.B. Prins, E. Bazelmans Cognitive behavioural therapies L.A. Jason, P.A. Fennell, R.R. Taylor (Eds.), Handbook of chronic fatigue syndrome, Wiley \& Sons, New York (2003)

E.S. Bordin The generalizability of the psychoanalytic concept of the working alliance Psychotherapy: Theory, Research \& Practice, 16 (1979), pp. 252-260

B.D. Castell, N. Kazantzis, R.E. Moss-Morris Cognitive behavioral therapy and graded exercise for chronic fatigue syndrome: a meta-analysis Clinical Psychology: Science and Practice, 18 (2011), pp. 311-324 
Heins, M.J., Knoop, H., Bleijenberg, G. The role of the therapeutic relationship in cognitive behaviour therapy for chronic fatigue syndrome. Behaviour Research and Therapy: 2013, 51(7), ) 368-376

M. Cella, D. Stahl, S.E. Reme, T. Chalder Therapist effects in routine psychotherapy practice: an account from chronic fatigue syndrome Psychotherapy Research, 21 (2011), pp. 168-178

D. Chambers, A.M. Bagnall, S. Hempel, C. Forbes Interventions for the treatment, management and rehabilitation of patients with chronic fatigue syndrome/myalgic encephalomyelitis: an updated systematic review Journal of the Royal Society of Medicine, 99 (2006), pp. 506-520

H.J. Cho, M. Hotopf, S. Wessely The placebo response in the treatment of chronic fatigue syndrome: a systematic review and meta-analysis Psychosomatic Medicine, 67 (2005), pp. 301-313

Y. Christley, T. Duffy, C.R. Martin A review of the definitional criteria for chronic fatigue syndrome Journal of Evaluation in Clinical Practice, 18 (2012)

D.A. Clark, A.T. Beck, B.A. Alford Scientific foundations of cognitive theory and therapy of depression John Wiley \& Sons Inc; US, Hoboken, NJ (1999)

M.J. Constantino, D.B. Arnkoff, C.R. Glass, R.M. Ametrano, J.Z. Smith Expectations

Journal of Clinical Psychology, 67 (2011), pp. 184-192

F.M. Dattilio, M.A. Hanna Collaboration in cognitive-behavioral therapy Journal of Clinical Psychology, 68 (2012), pp. 146-158

B. De Vree, S. Van der Werf, J.B. Prins, E. Bazelmans, J.H. vercoulen, P. Servaeset al. Meetinstrumenten bij chronische vermoeidheid [Measurement instruments in chronic fatigue] Gedragstherapie, 35 (2002), pp. 157-164

A. Deale, T. Chalder, S. Wessely Illness beliefs and treatment outcome in chronic fatigue syndrome Journal of Psychosomatic Research, 45 (1998), pp. 77-83

Z. Di Blasi, E. Harkness, E. Ernst, A. Georgiou, J. Kleijnen Influence of context effects on health outcomes: a systematic review Lancet, 357 (2001), pp. 757-762

C. Fluckiger, A.C. Del Re, B.E. Wampold, D. Symonds, A.O. Horvath How central is the alliance in psychotherapy? A multilevel longitudinal meta-analysis Journal of Counseling Psychology, 59 (2012), pp. 10-17

A.M. Fry, M. Martin Fatigue in the chronic fatigue syndrome: a cognitive phenomenon? Journal of Psychosomatic Research, 41 (1996), pp. 415-426

K. Fukuda, S.E. Straus, I. Hickie, M.C. Sharpe, J.G. Dobbins, A. Komaroff The chronic fatigue syndrome: a comprehensive approach to its definition and study. International Chronic Fatigue Syndrome Study Group Annals of Internal Medicine, 121 (1994), pp. 953959

J. Gaab Psychotherapie chronischer Erschöpfungszustände Psychotherapeut, 49 (2004), pp. 431-445

L. Gaston, L. Thompson, D. Gallagher, L.-G. Cournoyer, R. Gagnon Alliance, technique, and their interactions in predicting outcome of behavioral, cognitive, and brief dynamic therapy

Psychotherapy Research, 8 (1998), pp. 190-209

E. Godfrey, T. Chalder, L. Ridsdale, P. Seed, J. Ogden Investigating the active ingredients of cognitive behaviour therapy and counselling for patients with chronic fatigue in primary care: developing a new process measure to assess treatment fidelity and predict outcome British Journal of Clinical Psychology, 46 (2007), pp. 253-272

R.P. Greenberg, M.J. Constantino, N. Bruce Are patient expectations still relevant for psychotherapy process and outcome? Clinical Psychology Review, 26 (2006), pp. 657-678

R.L. Hatcher, A.W. Barends How a return to theory could help alliance research

Psychotherapy: Theory, Research, Practice, Training, 43 (2006)

Heins, M., Knoop, J., Burk, W., \& Bleijenberg, G. (2013). The process of cognitive behaviour therapy for chronic fatigue syndrome: Which changes in perpetuating cognitions and behaviour are related to a reduction in fatigue? (submitted for publication). 
Heins, M.J., Knoop, H., Bleijenberg, G. The role of the therapeutic relationship in cognitive behaviour therapy for chronic fatigue syndrome. Behaviour Research and Therapy: 2013, 51(7), ) 368-376

A. Hoffart, F.-M. Borge, H. Sexton, D.M. Clark, B.E. Wampold Psychotherapy for social phobia: how do alliance and cognitive process interact to produce outcome?

Psychotherapy Research, 22 (2011), pp. 82-94

A.O. Horvath, A.C. Del Re, C. Fluckiger, D. Symonds Alliance in individual psychotherapy Psychotherapy Special Issue: Evidence-based Psychotherapy Relationships, 48 (2011), pp. 9-16

A.O. Horvath, B. Symonds Relation between working alliance and outcome in psychotherapy: a meta-analysis Journal of Counseling Psychology, 38 (1991), pp. 139-149 H.M. Jacobs, A. Luttik, F.W. Touw-Otten, R.A. de Melker The sickness impact profile; results of an evaluation study of the Dutch version Ned Tijdschr Geneeskd, 134 (1990), pp. 19501954

S. Kean Virology. An indefatigable debate over chronic fatigue syndrome Science, 327 (2010), pp. 254-255

H. Knoop, G. Bleijenberg Het chronisch vermoeidheidssyndroom: behandelprotocol cognitieve gedragstherapie voor CVS Bohn, Stafleu en van Loghum, Houten (2010)

H. Knoop, G. Bleijenberg, M.F. Gielissen, J.W. van der Meer, P.D. White Is a full recovery possible after cognitive behavioural therapy for chronic fatigue syndrome? Psychotherapy and Psychosomatics, 76 (2007), pp. 171-176

D.J. Martin, J.P. Garske, M.K. Davis Relation of the therapeutic alliance with outcome and other variables: a meta-analytic review Journal of Consulting and Clinical Psychology, 68 (2000), pp. 438-450

J.C. Norcross Purposes, processes and products of the task force on empirically supported therapy relationships Psychotherapy: Theory, Research, Practice, Training, 38 (2001), pp. 345-356

J.C. Norcross, B.E. Wampold Evidence-based therapy relationships: research conclusions and clinical practices Psychotherapy, 48 (2011), pp. 98-102

W.E. Pollard, R.A. Bobbitt, M. Bergner, D.P. Martin, B.S. Gilson The Sickness Impact Profile: reliability of a health status measure Medical Care, 14 (1976), pp. 146-155

J.R. Price, E. Mitchell, E. Tidy, V. Hunot Cognitive behaviour therapy for chronic fatigue syndrome in adults Cochrane Database of Systematic Reviews (2008), p. CD001027

J.B. Prins, G. Bleijenberg, E. Bazelmans, L.D. Elving, T.M. de Boo, J.L. Severens et al. Cognitive behaviour therapy for chronic fatigue syndrome: a multicentre randomised controlled trial Lancet, 357 (2001), pp. 841-847

C. Ray, W. Weir, D. Stewart, P. Miller, G. Hyde Ways of coping with chronic fatigue syndrome: development of an illness management questionnaire Social Science \& Medicine, 37 (1993), pp. 385-391

W.C. Reeves, A. Lloyd, S.D. Vernon, N. Klimas, L.A. Jason, G. Bleijenberg et al. Identification of ambiguities in the 1994 chronic fatigue syndrome research case definition and recommendations for resolution BMC Health Services Research, 3 (2003), p. 25

A.L. Steward, R.D. Hays, J.E. Ware Jr. The MOS short form general health survey: reliability and validity in a patient population Medical Care, 26 (1998), pp. 724-735

N. Stinckens, A. Ulburghs, L. Claes De werkalliantie als sleutelelement in het therapiegebeuren: Meting met behulp van de WAV-12, de Nederlandstalige verkorte versie van de Working Alliance Inventory Tijdschrift Klinische Psychologie, 39 (2009), pp. 44-60

C. Surawy, A. Hackmann, K. Hawton, M. Sharpe Chronic fatigue syndrome: a cognitive approach Behaviour Research and Therapy, 33 (1995), pp. 535-544

J. Tee, N. Kazantzis Collaborative empiricism in cognitive therapy: a definition and theory for the relationship construct Clinical Psychology: Science and Practice, 18 (2011)

T.J. Tracey, A.M. Kokotovic Factor structure of the working alliance Inventory Psychological Assessment: A Journal of Consulting and Clinical Psychology, 1 (1989), pp. 207-210 
Heins, M.J., Knoop, H., Bleijenberg, G. The role of the therapeutic relationship in cognitive behaviour therapy for chronic fatigue syndrome. Behaviour Research and Therapy: 2013, 51(7), 368-376

P.G. Ulvenes, L. Berggraf, A. Hoffart, T.C. Stiles, M. Svartberg, L. McCullough et al. Different processes for different therapies: therapist actions, therapeutic bond, and outcome

Psychotherapy, 49 (2012), pp. 291-302

B. Van Houdenhove What is the aim of cognitive behaviour therapy in patients with chronic fatigue syndrome? Psychotherapy and Psychosomatics, 75 (2006), pp. 396-397

J.H. Vercoulen, M. Alberts, G. Bleijenberg De checklist individual strength (CIS) Gedragstherapie, 32 (1999), pp. 31-36

J.H. Vercoulen, C.M. Swanink, J.F. Fennis, J.M. Galama, J.W. van der Meer, G. Bleijenberg Dimensional assessment of chronic fatigue syndrome J Psychosom Res, 38 (1994), pp. 383-392

J.H. Vercoulen, C.M. Swanink, J.M. Galama, J.F. Fennis, P.J. Jongen, O.R. Hommes et al. The persistence of fatigue in chronic fatigue syndrome and multiple sclerosis: development of a model Journal of Psychosomatic Research, 45 (1998), pp. 507-517

B.E. Wampold, S.L. Budge The 2011 Leona Tyler Award Address: the relationship-And its relationship to the common and specific factors of psychotherapy The Counseling Psychologist, 40 (2012)

S.P. van der Werf, B. de Vree, M. Alberts, J.W.M. van der Meer, G. Bleijenberg Natural course and predicting self-reported improvement in patients with chronic fatigue syndrome with a relatively short illness duration Journal of Psychosomatic Research, 53 (2002), pp. 749-753

S. Wessely, A. David, S. Butler, T. Chalder Management of chronic (post-viral) fatigue syndrome The Journal of the Royal College of General Practitioners, 39 (1989), pp. 26-29

H.A. Westra, D.J.A. Dozois, M. Marcus Expectancy, homework compliance, and initial change in cognitive-behavioral therapy for anxiety Journal of Consulting and Clinical Psychology, 75 (2007), pp. 363-373

P. White, K. Goldsmith, A. Johnson, L. Potts, R. Walwyn, J. Decesare et al. Comparison of adaptive pacing therapy, cognitive behaviour therapy, graded exercise therapy, and specialist medical care for chronic fatigue syndrome (PACE): a randomised trial Lancet, 377 (2011), pp. 823-836

J.F. Wiborg, H. Knoop, L.E. Frank, G. Bleijenberg Towards an evidence-based treatment model for cognitive behavioral interventions focusing on chronic fatigue syndrome Journal of Psychosomatic Research, 72 (2012), pp. 399-404

J.F. Wiborg, H. Knoop, J.B. Prins, G. Bleijenberg Does a decrease in avoidance behavior and focusing on fatigue mediate the effect of cognitive behavior therapy for chronic fatigue syndrome? Journal of Psychosomatic Research, 70 (2011), pp. 306-310

J.F. Wiborg, H. Knoop, M. Stulemeijer, J.B. Prins, G. Bleijenberg

How does cognitive behaviour therapy reduce fatigue in patients with chronic fatigue syndrome? The role of physical activity Psychological Medicine, 40 (2010), pp. 1281-1287

J.F. Wiborg, H. Knoop, M. Wensing, G. Bleijenberg Therapist effects and the dissemination of cognitive behavior therapy for chronic fatigue syndrome in community-based mental health care Behaviour Research and Therapy, 50 (2012), pp. 393-39

Working group on CFS/ME (Chronic Fatigue Syndrome/Myalgic Encephalomyelitis) A report of the CFS/ME working group epartment of Health, London (2002) 
Heins, M.J., Knoop, H., Bleijenberg, G. The role of the therapeutic relationship in cognitive behaviour therapy for chronic fatigue syndrome. Behaviour Research and Therapy: 2013, 51(7), 368-376

\section{APPENDIX, FIGURES AND TABLES}

Fig. 1. Flow chart of the selection of the study participants

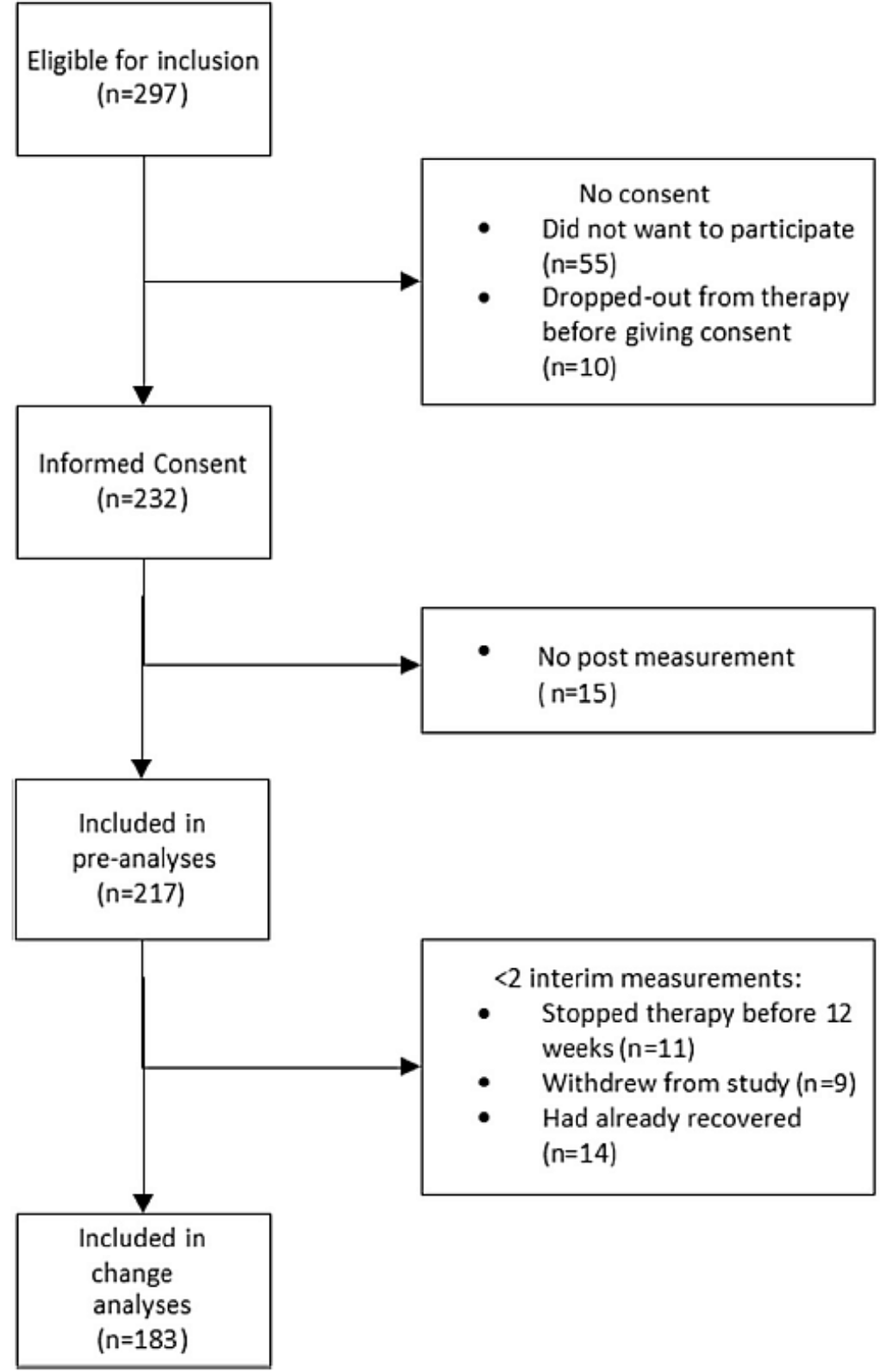


Heins, M.J., Knoop, H., Bleijenberg, G. The role of the therapeutic relationship in cognitive behaviour therapy for chronic fatigue syndrome. Behaviour Research and Therapy: 2013, 51(7), 368-376

Table 1

Baseline characteristics $(n=217)$.

\begin{tabular}{lc}
\hline & Mean (sd) \\
\hline Variable & \\
Age (years) & $37.8(11.2)$ \\
Female & $162(74.7 \%)$ \\
CIS fatigue & $49.7(5.4)$ \\
SIP total score & $1535(560)$ \\
No. CDC symptoms & $7(1.7)$ \\
Symptom durations (years) & $5.0(3.0-10.8)$ \\
Patient & \\
WAI-s Task & $16.2(2.5)$ \\
WAI-s Bond & $15.1(2.4)$ \\
WAI-s Goal & $16.7(2.4)$ \\
Expectations & $10.2(1.4)$ \\
Therapist & \\
WAI-s Task & $16.5(2.0)$ \\
WAI-s Bond & $16.4(1.8)$ \\
WAI-s Goal & $16.4(2.2)$ \\
Expectations & $85(73-93)$ \\
\hline
\end{tabular}

* Absolute number (percentage) are given. CIS fatigue = Checklist Individual strength; Outcome expectations = Therapy Outcome Expectations Questionnaire; SIP = Sickness Impact Profile; WAI-S=Working Alliance Inventory Short Form.

Table 2

Prediction models of baseline patient and therapist rated therapy relationship $(n=217)$.

\begin{tabular}{|c|c|c|c|c|c|c|c|c|c|}
\hline & \multicolumn{3}{|l|}{1} & \multicolumn{3}{|l|}{2} & \multicolumn{3}{|l|}{3} \\
\hline & $\mathrm{B}(\mathrm{se})$ & $t$ & $p$ & $\mathrm{~B}(\mathrm{se})$ & $t$ & $p$ & $\mathrm{~B}(\mathrm{se})$ & $t$ & $p$ \\
\hline \multicolumn{10}{|l|}{ Baseline } \\
\hline Fatigue pre & $0.44(0.18)$ & 2.45 & 0.06 & $0.51(0.16)$ & 3.20 & 0.01 & $0.48(0.16)$ & 3.00 & 0.02 \\
\hline Sex & $3.57(2.28)$ & 1.57 & 0.21 & $0.78(1.99)$ & 0.39 & 0.73 & $-0.03(1.98)$ & -0.01 & 0.99 \\
\hline Age (year) & $0.14(0.09)$ & 1.50 & 0.22 & $0.17(0.08)$ & 2.11 & 0.10 & $0.14(0.08)$ & 1.82 & 0.16 \\
\hline Phys attr & $0.20(0.35)$ & 0.57 & 0.63 & $-0.37(0.31)$ & -1.21 & 0.33 & $-0.41(0.30)$ & -1.34 & 0.28 \\
\hline SIP pre & $0.00(0.00)$ & 1.18 & 0.33 & $0.00(0.00)$ & 1.70 & 0.18 & $0.00(0.00)$ & 1.84 & 0.17 \\
\hline Duration (mth) & $0.14(0.09)$ & 1.57 & 0.21 & $0.11(0.08)$ & 1.33 & 0.28 & $0.07(0.08)$ & 0.93 & 0.47 \\
\hline \multicolumn{10}{|l|}{ Patient } \\
\hline WAI-s Task & & & & $-1.40(0.50))$ & -2.80 & 0.03 & $-1.40(0.50)$ & -2.79 & 0.03 \\
\hline WAI-s Bond & & & & $0.10(0.42)$ & 0.24 & 0.83 & $0.24(0.420$ & 0.57 & 0.63 \\
\hline WAI-s Goal & & & & $0.47(0.54)$ & 0.87 & 0.48 & $0.40(0.54)$ & 0.75 & 0.53 \\
\hline Expectations & & & & $-4.28(0.67)$ & -6.39 & $<0.001$ & $-3.96(0.69)$ & -5.75 & $<0.001$ \\
\hline \multicolumn{10}{|l|}{ Therapist } \\
\hline WAI-s Task & & & & & & & $1.24(0.67)$ & 1.87 & 0.15 \\
\hline WAI-s Bond & & & & & & & $-0.47(0.58)$ & -0.80 & 0.52 \\
\hline WAI-s Goal & & & & & & & $-0.73(0.56)$ & -1.31 & 0.28 \\
\hline Expectations & & & & & & & $-0.16(0.060)$ & -2.52 & 0.03 \\
\hline (Constant) & $-7.47(10.36)$ & -0.72 & & $52.59(11.57)$ & 4.55 & & $63.04(12.54)$ & 5.03 & \\
\hline$R^{2 \mathrm{a}}$ & 0.078 & & 0.03 & 0.330 & & $<0.001$ & 0.365 & & 0.09 \\
\hline
\end{tabular}

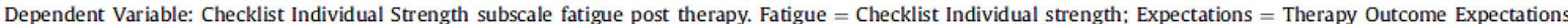

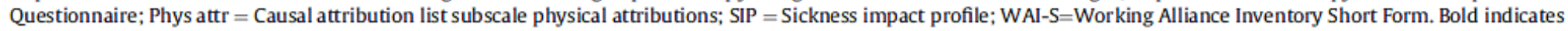
$p<0.05$.

${ }^{\text {a }} R^{2}$ and $t$-value for $F$-test of change. 
Heins, M.J., Knoop, H., Bleijenberg, G. The role of the therapeutic relationship in cognitive behaviour therapy for chronic fatigue syndrome. Behaviour Research and Therapy: 2013, 51(7), 368-376

Table 3a

Prediction models of change in patient and therapist rated therapy relationship early in therapy $(n=183)$.

\begin{tabular}{|c|c|c|c|c|c|c|c|c|c|}
\hline & \multicolumn{3}{|l|}{1} & \multicolumn{3}{|l|}{2} & \multicolumn{3}{|l|}{3} \\
\hline & $\mathrm{B}(\mathrm{se})$ & $t$ & $p$ & $\mathrm{~B}(\mathrm{se})$ & $t$ & $p$ & $\mathrm{~B}(\mathrm{se})$ & $t$ & $p$ \\
\hline \multicolumn{10}{|l|}{ Baseline } \\
\hline Fatigue pre & $0.46(-0.16)$ & 2.80 & 0.02 & $0.41(0.16)$ & 2.56 & 0.04 & $0.36(0.16)$ & 2.21 & 0.08 \\
\hline WAI-s Task pt & $-1.17(0.52)$ & -2.25 & 0.08 & $-0.62(0.68)$ & -0.92 & 0.54 & $-0.81(0.68)$ & -1.19 & 0.44 \\
\hline WAI-s Bond $\mathrm{pt}$ & $0.07(0.44)$ & 0.17 & 0.91 & $-0.36(0.50)$ & -0.72 & 0.63 & $-0.30(0.50)$ & -0.61 & 0.68 \\
\hline WAI-s Goal pt & $0.35(0.56)$ & 0.62 & 0.68 & $0.79(0.81)$ & 0.97 & 0.54 & $0.95(0.82)$ & 1.17 & 0.43 \\
\hline Expectations pt & $-3.32(0.71)$ & -4.70 & $<0.001$ & $-4.60(0.80)$ & -5.73 & $<0.001$ & $-4.24(0.84)$ & -5.07 & $<0.001$ \\
\hline WAI-s Task ther & $1.37(0.70)$ & 1.95 & 0.12 & $1.46(0.68)$ & 2.14 & 0.08 & $0.24(0.97)$ & 0.25 & 0.87 \\
\hline WAI-s Bond ther & $-0.45(0.62)$ & -0.73 & 0.63 & $-0.51(0.60)$ & -0.86 & 0.56 & $-0.41(0.79)$ & -0.52 & 0.67 \\
\hline WAI-s Goal ther & $-0.75(0.60)$ & -1.24 & 0.42 & $-0.85(0.60)$ & -1.43 & 0.32 & $0.06(0.82)$ & 0.08 & 0.96 \\
\hline $\begin{array}{l}\text { Expectations ther } \\
\text { Patient }\end{array}$ & $-0.24(0.06)$ & -3.79 & $<0.001$ & $-0.24(0.06)$ & -3.86 & $<0.001$ & $-0.23(0.07)$ & -3.45 & 0.004 \\
\hline$\Delta$ WAI-s Task & & & & $0.40(0.57)$ & 0.71 & 0.63 & $0.33(0.57)$ & 0.58 & 0.68 \\
\hline$\Delta$ WAI-s Bond & & & & $-0.78(0.45)$ & -1.73 & 0.21 & $-0.70(0.46)$ & -1.52 & 0.29 \\
\hline$\Delta$ WAI-s Goal & & & & $0.35(0.71)$ & 0.49 & 0.70 & $0.39(0.72)$ & 0.55 & 0.68 \\
\hline $\begin{array}{l}\Delta \text { Expectations } \\
\text { Therapist }\end{array}$ & & & & $-3.02(0.83)$ & -3.65 & $<0.001$ & $-2.92(0.84)$ & -3.48 & 0.004 \\
\hline$\Delta$ WAI-s Task & & & & & & & $-1.63(0.83)$ & -1.96 & 0.12 \\
\hline$\Delta$ WAI-s Bond & & & & & & & $0.01(0.79)$ & 0.02 & 0.99 \\
\hline$\Delta$ WAI-s Goal & & & & & & & $0.93(0.70)$ & 132 & 0.38 \\
\hline$\Delta$ Expectations & & & & & & & $0.05(0.05)$ & 0.91 & 0.54 \\
\hline constant & $67.08(11.99)$ & 5.60 & & $74.39(11.81)$ & 6.30 & & $75.46(12.44)$ & 6.07 & \\
\hline$R^{2}$ & 0.323 & & $<0.001$ & 0.386 & & 0.004 & 0.403 & & 0.47 \\
\hline
\end{tabular}

Dependent Variable: Checklist Individual Strength subscale fatigue post therapy. Fatigue = Checklist Individual strength; Expectations $=$ Therapy Outcome Expectations Questionnaire; WAI-S=Working Alliance Inventory Short Form.

$R^{2}$ and $t$-value for $F$-test of change. Bold indicates $p<0.05$.

Table 3b

Prediction models of change in patient and therapist rated therapy relationship mid-therapy $(n=183)$.

\begin{tabular}{|c|c|c|c|c|c|c|c|c|c|}
\hline & \multicolumn{3}{|l|}{1} & \multicolumn{3}{|l|}{2} & \multicolumn{3}{|l|}{3} \\
\hline & $\mathrm{B}(\mathrm{se})$ & $t$ & $p$ & $\mathrm{~B}(\mathrm{se})$ & $t$ & $p$ & $\mathrm{~B}(\mathrm{se})$ & $t$ & $p$ \\
\hline \multicolumn{10}{|l|}{ Baseline } \\
\hline Fatigue pre & $0.23(0.19)$ & 1.23 & 0.39 & $0.12(0.18)$ & 0.68 & 0.57 & $0.14(0.19)$ & 0.74 & 0.57 \\
\hline WAI-s Task pt int 1 & $-0.65(0.56)$ & -1.16 & 0.40 & $-0.85(0.72)$ & -1.18 & 0.40 & $-0.93(0.73)$ & -1.28 & 0.39 \\
\hline WAI-s Bond pt int1 & $-0.41(0.44)$ & -0.94 & 0.51 & $0.01(0.48)$ & 0.02 & 0.98 & $-0.20(0.49)$ & -0.41 & 0.77 \\
\hline WAI-s Goal pt int 1 & $0.79(0.76)$ & 1.04 & 0.45 & $0.52(0.93)$ & 0.56 & 0.68 & $0.65(0.94)$ & 0.69 & 0.59 \\
\hline Expectations pt int 1 & $-3.35(0.78)$ & -4.31 & 0.001 & $-3.65(0.87)$ & -4.22 & 0.001 & $-3.02(0.91)$ & -3.31 & 0.008 \\
\hline WAI-s Task ther int1 & $-1.67(0.91)$ & -1.84 & 0.25 & $-1.78(0.86)$ & -2.06 & 0.19 & $-2.16(1.14)$ & -1.89 & 0.23 \\
\hline WAI-s Bond ther int1 & $-0.12(0.78)$ & -0.15 & 0.95 & $0.04(0.73)$ & 0.05 & 0.98 & $0.77(0.86)$ & 0.91 & 0.51 \\
\hline WAI-s Goal ther int1 & $1.35(0.78)$ & 1.74 & 0.26 & $1.14(0.73)$ & 1.56 & 0.32 & $1.25(1.00)$ & 1.25 & 0.38 \\
\hline $\begin{array}{l}\text { Expectations ther int } 1 \\
\text { Patient }\end{array}$ & $-0.06(0.05)$ & -122 & 0.39 & $-0.07(0.05)$ & -1.41 & 0.35 & $-0.20(0.08)$ & -2.49 & 0.06 \\
\hline$\Delta$ WAI-s Task & & & & $-1.29(0.67)$ & -1.91 & 0.23 & $-1.10(0.67)$ & -1.64 & 0.30 \\
\hline$\Delta$ WAI-s Bond & & & & $0.77(0.51)$ & 1.50 & 0.34 & $0.66(0.51)$ & 1.31 & 0.38 \\
\hline$\Delta$ WAI-s Goal & & & & $-0.58(0.66)$ & -0.88 & 0.51 & $-0.59(0.67)$ & -0.87 & 0.51 \\
\hline$\Delta$ Expectations & & & & $-2.01(0.74)$ & -2.71 & 0.06 & $-1.27(0.81)$ & -1.57 & 0.32 \\
\hline \multicolumn{10}{|l|}{ Therapist } \\
\hline$\Delta$ WAI-s Task & & & & & & & $-0.17(0.90)$ & -0.19 & 0.94 \\
\hline$\Delta$ WAI-s Bond & & & & & & & $0.93(0.86)$ & 1.08 & 0.44 \\
\hline$\Delta$ WAI-s Goal & & & & & & & $-0.11(0.82)$ & -0.13 & 0.95 \\
\hline$\Delta$ Expectations & & & & & & & $-0.18(0.09)$ & -2.08 & 0.19 \\
\hline constant & $65.41(13.98)$ & 4.68 & & $77.69(13.77)$ & 5.64 & & $76.42(14.35)$ & 5.33 & \\
\hline$R^{2}$ & 0.277 & & 0.001 & 0.380 & & 0.001 & 0.340 & & 0.35 \\
\hline
\end{tabular}

Dependent Variable: Checklist Individual Strength subscale fatigue post therapy. Fatigue = Checklist Individual strength; Expectations = Therapy Outcome Expectations Questionnaire; WAI-S=Working Alliance Inventory Short Form.

$R^{2}$ and $t$-value for $F$-test of change. Bold indicates $p<0.05$. 
Heins, M.J., Knoop, H., Bleijenberg, G. The role of the therapeutic relationship in cognitive behaviour therapy for chronic fatigue syndrome. Behaviour Research and Therapy: 2013, 51(7), 368-376

Table 4

Final model of specific and non-specific factors $(n=183)$.

\begin{tabular}{lrrc}
\hline & $\mathrm{B}(\mathrm{se})$ & \multicolumn{1}{c}{$t$} & \multicolumn{1}{c}{$p$} \\
\hline Fatigue pre & $0.88(0.13)$ & 6.96 & $<0.001$ \\
Task pt pre & $-0.54(0.28)$ & -1.91 & 0.09 \\
Expect pt pre & $-0.70(0.63)$ & -1.11 & 0.38 \\
Expect ther pre & $-0.11(0.04)$ & -2.71 & 0.02 \\
$\Delta$ Expect pt 1 & $-0.38(0.66)$ & -0.57 & 0.69 \\
$\Delta$ Expect pt 2 & $-0.18(0.51)$ & -0.36 & 0.77 \\
$\Delta$ Focus & $0.15(0.08)$ & 1.96 & 0.09 \\
$\Delta$ Control & $-0.82(0.17)$ & -4.76 & $<0.001$ \\
$\Delta$ Perc activity & $0.55(0.14)$ & 3.97 & $<0.001$ \\
$\Delta$ Phys funct & $-0.19(0.04)$ & -5.02 & $<0.001$ \\
Constant & & & \\
\hline
\end{tabular}

Dependent Variable: Checklist Individual Strength subscale fatigue post therapy. Control = Self-efficacy Scale; Fatigue = Checklist Individual strength; Expectations $=$ Therapy Outcome Expectations Questionnaire; Focus = Illness Management Questionnaire subscale focusing on symptoms; Perc activity $=$ Checklist Individual Strength subscale physical activity; Phys funct $=$ MOS SF-36 subscale physical functioning WAI-S=Working Alliance Inventory Short Form. Bold indicates $p<0.05$. 
The process of cognitive behaviour therapy for chronic fatigue syndrome: Which changes in perpetuating cognitions and behaviour are related to a reduction in fatigue?

Running head: The process of CBT for CFS

Expert Centre for Chronic Fatigue, Radboud University Nijmegen Medical Centre, The Netherlands Marianne J Heins MSc, Hans Knoop PhD, Gijs Bleijenberg Phd

Correspondence to:

Marianne Heins

Radboud University Nijmegen Medical Centre

Expert Centre for Chronic Fatigue, 4628

PO Box 9101

6500 HB Nijmegen

The Netherlands

Tel +31243610046

Fax +31243610041

E-mail: m.heins@nkcv.umcn.nl

Word count: 3971 


\section{Abstract}

Objective: Cognitive behaviour therapy (CBT) can significantly reduce fatigue in chronic fatigue syndrome (CFS), but little is known about the process of change taking place during CBT. Based on a recent treatment model (Wiborg et al. J Psych Res 2012), we examined how changes in cognitions and behaviour are related to the decrease in fatigue. Methods: We included 183 patients meeting the US Centers for Disease Control criteria for CFS, aged 18 to 65 years, starting CBT. We measured fatigue and possible process variables before, after 6,12 and 18 weeks, and at the end of treatment. Possible process variables were sense of control over fatigue, focusing on symptoms, self-reported physical functioning, perceived physical activity and actual (actigraphic) physical activity. A structural equation model was built, explaining changes in fatigue during therapy by changes in proposed process variables. Results: Despite large individual variation in the timing of change during CBT for CFS, increases in the sense of control over fatigue, perceived activity and self-reported physical functioning, and decreases in focusing on symptoms explained 49 to $67 \%$ of the decrease in fatigue. An increase in actual physical activity was no process variable. Conclusion: A change in cognitive factors is related to the decrease in fatigue during CBT for CFS. The pattern of change varies considerably between patients, but changes in process variables and fatigue occur mostly in the same period.

Keywords: Chronic Fatigue Syndrome; Cognitive Behaviour Therapy; Process; Change; Sense of control; Focusing 


\section{Introduction}

Chronic fatigue syndrome (CFS) is diagnosed when someone experiences medically unexplained, severe fatigue that has been present for at least six months and results in severe impairment in daily functioning $(1,2)$. Several cognitive behavioural models of CFS exist, which try to explain the perpetuation of symptoms in CFS patients (3-6). All start with a period of severe fatigue that patients, rightfully or not, often attribute to a physical illness. In order to recover, patients often rest and become less active. Most models assume that because of this inactivity, patients' physical condition will decline and they will become increasingly sensitive to fatigue. In one of these models, that of Vercoulen et al. (7), a low level of physical activity, a low sense of control over fatigue, and a high level of focusing on symptoms explain the perpetuation of fatigue and impairment. The low level of physical activity is related to somatic attributions. Although recent studies have shed more light on the perpetuating factors in CFS, this is still the only model that simultaneously tested the influence of several perpetuating factors. Based on these models, several protocols for CBT were developed (811) that lead to a significant reduction of fatigue and impairment $(12,13)$. Although protocols differ, they all encompass a stepwise increase in physical activity and challenging of dysfunctional fatiguerelated beliefs.

Recently, Wiborg et al. developed a comprehensive treatment model of CBT for CFS (14), based on the model of Vercoulen et al. (7). For their analyses they used data from previous randomised controlled trials (RCTs) testing the efficacy of CBT for CFS. Wiborg et al. first tested for each variable in the model of Vercoulen whether it was a mediator of CBT for CFS, i.e. whether it changed more in patients receiving $\mathrm{CBT}$ than in the control group and whether this change was related to treatment outcome. Two adaptations were made to the original variables: Somatic attributions were not analysed, as previous research had shown that somatic attributions do not change during treatment (9), so they could never be a mediator of CBT; perceived problems with activity were analysed, rather than actual activity assessed with actigraphy, as previous research had shown that actual activity or physical fitness do not mediate the effect of CBT for CFS (15) and other behavioural interventions for 
chronic fatigue $(16,17)$. In the final model, the decrease in fatigue is explained by an increased sense of control over fatigue, an increase in perceived activity, and improved physical functioning.

There are three limitations to the model of Wiborg et al. First, the model only relied on measurements before and after therapy and did not study the process of change during therapy. We therefore do not know whether changes in mediating variables indeed precede changes in fatigue. This makes it impossible to draw causal conclusions. Furthermore, temporary changes, which may catalyse other change processes, will go unnoticed. For example, a permanent change in actual physical activity does not mediate the treatment effect, but a temporary increase in activity might lead to changes in cognitions, which then lead to a decrease in fatigue. Second, focusing on symptoms was measured with the subscale 'somatic complaints' of the Symptom Checklist 90. As the authors argued themselves, this questionnaire may have been unsuitable. Third, the model was tested in an RCT of a minimal intervention based on CBT for CFS (18). Patients received a self-help booklet explaining the different components of CBT. In addition, they had regular e-mail contact with a therapist. Tummers et al. showed that fatigue and impairment decrease significantly when patients follow additional CBT after such a minimal intervention (19). So individual CBT may have a more elaborate mechanism of change than a minimal intervention.

We built on the treatment model of Wiborg et al. by studying the change in different process variables during individual CBT. In figure 1 all proposed process variables are depicted. First of all, we selected the variables of the model of Wiborg et al., i.e. sense of control over fatigue, perceived activity and self-reported physical functioning. We added focusing on symptoms because this variable mediated the effect of CBT for CFS in an earlier RCT (20) and may have been measured inadequately in the study of Wiborg et al. These four variables had thus been proven to mediate the effect of CBT for CFS in formal mediation analyses of RCTs. Although actual physical activity was previously discarded as a mediator of CBT for CFS (15), we included this variable because a temporary change in actual physical activity might elicit other changes. 


\section{Methods}

\section{Patients}

Consecutively referred patients who started CBT at our tertiary CFS specialist care centre between April 2008 and September 2010 were eligible to participate if they:

- Met the criteria for CFS as stated by the US Centers for Disease Control and Prevention $(1,2)$. Severe fatigue was defined as a score of $\geq 35$ on the Checklist Individual Strength (CIS) subscale fatigue severity (21), and severe functional impairment was defined as a total score $\geq 700$ on the Sickness Impact Profile (SIP) (22-25). If a consultant or primary care physician had not ruled out any somatic disorder that excludes the diagnosis of CFS following the Fukuda criteria (1), patients first received a standard medical examination at the outpatient clinic of our department of internal medicine.

- Were between 18 and 65 years old

- Were able to read and write Dutch.

Patients were excluded if:

- They were currently applying for a disability claim until their application was completed, as this has previously been found to negatively influence treatment outcome (26).

The study was carried out in accordance with The Code of Ethics of the World Medical Association (Declaration of Helsinki) for experiments involving humans http://www.wma.net/en/30publications/10policies/b3/index.html; Uniform Requirements for manuscripts submitted to Biomedical journals http://www.icmje.org. It was approved by the ethical committee of the Radboud University Nijmegen Medical Centre. All participants gave written informed consent before participation. 


\section{CBT protocol}

At the start of therapy patients define concrete personal goals, usually including resumption of work, hobbies and other activities that imply recovery for the patient. Recovery, i.e. no longer being severely fatigued and impaired, is according to this protocol the highest goal to strive for in therapy. Patients are asked to regulate bedtimes and stop sleeping during the day, to stop possible disruption of the circadian rhythm. During the sessions, the therapist challenges non-accepting and catastrophising thoughts that inhibit adequate coping, and patients learn to distract their attention from their fatigue. Two groups of patients are discerned: Relatively active patients, characterised by bursts of activity followed by prolonged periods of rest, and low active patients, who score below the mean of CFS patients on at least $90 \%$ of the days during actigraphic measurement (27). Relatively active patients first spread their activities more evenly across the day. Low active patients start with a graded activity program immediately after the initial cognitive interventions. This activity program consists of daily walking or cycling, which is gradually increased. The increase in activity is not determined by the level of symptoms, but is time contingent. When patients succeed in increasing their physical activity, they start to increase social and mental activities. In the last phase of therapy, patients work systematically towards reaching their goals and experiment with fluctuating their bedtimes and activity level again. They are encouraged to perceive feelings of fatigue as a normal part of an active and healthy life and stop labelling themselves as a CFS patient $(8,28)$

\section{Measurements}

Besides the regular pre- and post therapy measurements we planned three interim measurements at 6,12 and 18 weeks after the start of therapy. Patients received the questionnaires from the researcher and were told that their answers would not be communicated with their therapist. This was also mentioned in the written study information and the written instructions accompanying the questionnaires.

\section{Fatigue}


Fatigue was assessed with the subscale fatigue severity of the Checklist Individual Strength (CIS) (21), indicating the level of fatigue in the previous two weeks, measured with eight items on a sevenpoint scale (range $8-56$ ). A score of 35 or higher (i.e. 2 standard deviations above the mean of a healthy control group) indicates severe fatigue. Cronbach's alpha ranges from .83 to $.92(7,29)$.

\section{Process variables}

\section{Sense of control over fatigue}

Sense of control over fatigue was assessed with the Self-Efficacy Scale (SES)(30, 31). This scale consists of five items (examples: Do you think you can influence your fatigue?; No matter what I do, I can't change my fatigue (reverse scoring); I think I could positively influence my fatigue). Items are scored on a 5-point Likert scale ranging from 'No, I am convinced that is not true' to 'Yes, I am convinced that is true'. The total score ranges from 5 to 25 , with higher scores indicating a higher sense of control over fatigue. The internal consistency of this scale ranges between .68 and .77 (30, 31).

\section{Focusing on symptoms}

Focusing on symptoms was measured with the subscale 'focusing on symptoms' of the Illness Management Questionnaire (32). This questionnaire measures the patient's approach to fatigue in the last 6 months, but for this study we changed this to the last few weeks. The subscale consists of 9 items measured on a 6-point Likert-scale (ranging from 'never' to 'always'). Cronbach's alpha is 0.88 (32).

\section{Actual physical activity}

Actual physical activity was measured using an actometer, a motion sensing device, worn around the ankle (27). The mean number of accelerations was calculated automatically every 5 minutes, based on which a mean daily activity score was calculated. Activity was registered during 12 days both before and after treatment. During treatment, actual physical activity was registered during 4 
complete days to limit the burden for participants. Data were visually checked to ensure that the device had worked properly and to check compliance. Days on which the device was worn for less than 22 hours were excluded.

\section{Perceived physical activity}

Perceived physical activity was measured with an adapted version of the subscale physical activity of the Checklist Individual Strength (21). This subscale indicates the perceived level of physical activity in the previous two weeks, measured with three items (I am physically very active (reverse scoring); I am physically not very active; My level of physical activity is low). These items are measured on a seven point scale ranging from 'Yes, that is true' to 'No, that is not true'. Scores range from $3-21$, with higher scores indicating a lower level of physical activity. The mean (SD) of the subscale in 915 untreated CFS patients was 18.0 (4.24) and Cronbach's alpha was .81.

\section{Physical functioning}

Limitations in self-reported physical functioning were measured with the corresponding subscale of the Medical Outcomes Survey SF36 (33). Scores range from 0 (maximum limitations) to 100 (no limitations). The Cronbach's alpha of the subscale of the Dutch version is .92 (34).

\section{Therapy components}

At the end of therapy we asked patients which therapy components had contributed most to their reduction in fatigue. They could select 13 different components (setting goals, helping thoughts, focusing attention away from fatigue, changing your sleep-wake pattern, spreading activities more evenly, increasing physical activity, getting back to work, communicating differently with significant others with respect to fatigue, increasing mental activity, increasing social activity, reaching your goals, no longer seeing yourself as a patient, solving future problems with fatigue).

\section{Statistics}


First, we determined the size of change in fatigue during CBT (uncontrolled Cohen's d) in our participants. We then assessed whether it was within the confines of a prior statistical benchmark of RCTs testing the efficacy of CBT for CFS (35).

Our goal was to determine how the pattern of change in fatigue during CBT was related to the pattern of change in process variables. To get a first visual impression, patients were divided into response groups, based on their levels of fatigue at the different measurements: fast responders (no severe fatigue at the first interim measurement and post therapy); mid-term responders (severe fatigue at the first interim measurement, but no longer at the second interim measurement and post therapy); slow responders (severe fatigue at the first and second interim measurement, but no longer post therapy); very slow responders (severe fatigue at the first, second and third interim measurement, but no longer post therapy); non responders (severe fatigue post therapy). Severe fatigue was defined as a score $\geq 35$ on the subscale fatigue of the CIS. Mean values of the potential process variables at the different measurements were calculated and depicted for each of these response groups.

We then tested statistically whether changes in fatigue and changes in process variables were related, using a structural equation model (see figure 2). The maximum likelihood method of estimation was used to estimate unknown regression parameters. Missing values were imputed using multiple imputation with 20 replications. The assessment of model fit was based on three goodness-of-fit indices: Chi square test, Bentler comparative fit index (CFI)(36) and the root mean square error of approximation (RMSEA) value (37). The CFI value indicates the proportion in the improvement of the overall fit of the specified model relative to an independence model in which the variables are assumed to be uncorrelated (38). The RMSEA fit index is an exact fit in which the null hypothesis states that the model corresponds to the data (RMSEA $=0.00)$. Data were considered to fit the model when Chi square statistics were non-significant ( $p>0.05$ ), RMSEA was up to 0.10 and CFI was above $.95(37,39)$. Non-significant chi square statistics imply that the specified model can be 
seen as acceptable, while the hypothesis that the observed covariance matrix is not different to the predicted covariance matrix by the specified model has not been rejected.

Data analysis was performed using SPSS 16.0 for Windows $^{\circledR}$ and MPlus ${ }^{\circledR}$ version 6.11 . Mean/standard deviation or median/interquartile range were calculated for normally distributed and skewed data respectively. A p-value $<0.05$ was considered statistically significant.

\section{Results}

\section{Patients}

In figure 3 a flow chart depicting the selection process is presented. Between April 2008 and December 2010, 297 CFS patients meeting all inclusion criteria started CBT. Fifty-five refused to participate; the most frequent reason given was the perceived effort of having to do extra assessments during treatment. Ten patients decided not to start with therapy in the first session, before informed consent was given. Of the 232 patients who gave written informed consent, 192 completed at least two interim measurements during the study: Fourteen decided to stop therapy before 12 weeks, 14 no longer experienced severe fatigue (fatigue $<35$ ) and therapist and patient agreed to stop, and 12 patients did no longer want to participate in the study. At the end of treatment, 9 patients did not fill in the questionnaires. So data of 183 patients could be included in the analyses. In 49 of them one or more variables were missing, which were imputed using multiple imputation with 20 replications. Seventy-five percent of them were female and their mean age was 38.2 ( $S D=11.5$ ). Mean CIS fatigue was 49.8 ( $S D=5.2$ ), mean SIP 1543 (SD 553) and the mean number of accompanying symptoms from the CDC definition was 7.05 (1.69). Thirty-eight (21\%) had a low activity pattern.

\section{Treatment outcome}


We compared the change in fatigue during our intervention to a statistical benchmark of CBT for CFS. The uncontrolled Cohen's d (40) for the change in fatigue severity during CBT was 2.11. This is higher than the statistical benchmark of CBT for CFS (1.44, 95\% Cl 0,97-1.89) (35). Post-hoc analyses suggested that patients participating in the study had a better treatment outcome than those who refused to participate and had data on post-treatment available $(n=41$, post treatment CIS subscale fatigue 27.5 (13.9) in participants vs. 36.0 (15.0) in non-participants: $d f=257 ; t=3.56 ; p<0.001)$.

\section{Response groups}

Based on their level of fatigue at the different measurements, 42 patients were classified as fastresponders (fatigue $<35$ at first interim measurement), 44 as mid-term responders (fatigue $<35$ at second interim measurement), 17 as slow-responders (fatigue $<35$ at third interim measurement), 24 as very slow responders (fatigue $<35$ at post measurement) and 52 as non-responders (fatigue $>=$ 35 at post measurement). We combined the slow and very-slow responders into one group of slow responders to obtain four groups of sufficient sample size (see figure 4). Because we combined the slow and very slow responders, we also combined the change scores between interim measurement 2 and the post measurement.

\section{Process variables related to change in fatigue}

Mean values of the potential process variables in the different response groups during therapy are depicted in figure 4. At post treatment, non-responders differed significantly from the other groups on all process variables.

Results of the structural equation model are given in table 1 . Model fit of the imputed model was excellent: $\mathrm{X}^{2}=7.19 ; \mathrm{df}=15 ; \mathrm{P}=0.95, \mathrm{CFI}=1.00$ and RMSEA $=0.00(90 \% \mathrm{Cl} 0.00-0.00)$. The model showed that in all three periods a decrease in fatigue was related to an improvement in sense of control over fatigue, physical functioning, perceived activity and focusing on symptoms in the same period. Increases in physical functioning in the first period also predicted decreases in fatigue in the second period. In none of the periods was the change in actual physical activity related to a decrease in 
fatigue. Changes in these process variables explained $49 \%$ of the change in fatigue in the first period, $50 \%$ in the second and $67 \%$ in the third period.

\section{What do patients themselves see as most helpful?}

At the end of therapy, we also asked patients what in their opinion had contributed most to the reduction in fatigue. The most frequently given answers were: changing your sleep-wake rhythm (72\%), getting back to work (65\%), increasing physical activity (60\%), and helping thoughts (57\%).

\section{Discussion}

This is the first time the process of change during CBT for CFS has been studied. We found a large variation in the time it takes to normalise fatigue. In around a quarter of the patients fatigue is already within normal limits 6 weeks after the start of CBT. Others are still severely fatigued after 18 weeks of therapy, but fatigue normalises thereafter. Anderson et al. found a similar variation in outpatient psychotherapy (41), but they studied a variety of diagnoses and therapeutic orientations. Despite this individual variation, we were able to discern four groups of patients (fast, mid-term, slow and non-responders) who show discernible patterns of change in fatigue and process variables.

During the entire therapy, a decrease in fatigue is accompanied by an increase in perceived activity, physical functioning and sense of control over fatigue together with a decrease in focusing on symptoms. This supports the importance of changes in cognitive factors in CBT for CFS. An increase in actual physical activity is not related to a decrease in fatigue. In the early phases of treatment, even patients who remain severely fatigued after treatment increase their level of physical activity. So an increase in physical activity per se does not seem sufficient to reduce fatigue. The increase in physical activity may merely catalyse the change process because patients experience that they can be more active despite being fatigued, which possibly facilitates a greater sense of control over fatigue and perhaps also leads to a decrease in focusing on symptoms. If such a change in cognitive processes does not occur, we could hypothesise, then patients do not experience a 
decrease in fatigue and fall back in their old patterns and levels of physical activity, even though their level of physical activity increased temporarily. Indeed, patients who remained severely fatigued did not retain their initial increases in activity; at the end of the treatment they were significantly less physically active, as measured by actigraphy, than the patients who had responded to treatment.

Although a gradual increase in actual physical activity does not mediate the treatment effect of CBT for CFS (15), modification of the physical activity pattern may play a role in subgroups of patients. Some high active patients may experience less fatigue by reducing activity and an increase in physical activity may be necessary in some low active patients. Future studies could experiment with various levels of activities and simultaneously monitor patients' change in fatigue. With initial increases in activity, patients may realise that they can be active in certain ways without increasing fatigue.

Changes in process variables were mostly related to changes in fatigue in the same period. As our study did not include a control group, we cannot say whether changes in process variables caused the decrease in fatigue, or vice versa. A decrease in fatigue may have permitted patients to be more active, may have given them a feeling of control over their fatigue and may have decreased their focus on fatigue. For most of the process variables we tested, previous mediation analysis of RCTs had proven that they mediated the effect of CBT for CFS $(14,20)$. Probably, the relationship between process variables and fatigue is not a simple cause-effect relationship, but a complex feedback process in which changes in cognitions lead to a decrease in fatigue, which leads to a further changes in cognitions and so on. Such a reciprocal relationship has previously been described for the natural course of CFS (42).

The process variables we examined indeed play an important role in CBT for CFS, as changes in these variables explained more than half of the changes in fatigue. But this leaves almost another half to be explained by other variables. One of them may be high standards that patients set for themselves, i.e. perfectionism $(3,4,43)$. These may lead to the bursts of activity that are 
characteristic for relatively active patients. A second candidate may be expectations of future performance. When patients already expect symptoms before starting a physical activity, they may also focus on possible feelings of fatigue, which will make them more sensitive to fatigue (5). Another candidate is the qualitative experience of fatigue. When asked to describe their fatigue, CFS patients use more negative adjectives than healthy persons $(9,44)$. A change in the negative representations of fatigue also mediates the effect of CBT for chronic fatigue in multiple sclerosis (45). Regulation of the sleep wake rhythm may also help reducing fatigue. In fact this was the therapy component patients most often mentioned as being helpful.

The relatively small number of patients in our study limited our analytic possibilities. We could only test a relatively simple path analysis model, whereas it would certainly have been interesting to use more sophisticated models, such as latent difference score models (46), or include factors that may possibly affect the process of change. As this is the first process study in CBT for CFS, we consider this to be an exploratory study and chose not to apply corrections for multiple testing.

A relatively large percentage of the patients starting CBT refused to participate in the study (19\%), mainly because the perceived effort of extra assessments during treatment. The patients who participated in our study may be those who were most motivated for the treatment, which may have led to a selection bias. The post-hoc analyses reported in the result section indeed suggest that treatment outcome in study participants was better than in patients who refused to participate. However, it is unlikely that the process of change was entirely different in those who refused to participate.

Concluding, a temporary increase in physical activity does not seem to be related to changes in fatigue during CBT for CFS. A change in cognitive factors such as the sense of control over fatigue and focusing on symptoms, and perceived activity and physical functioning seem related to the decrease in fatigue. The pattern of change in these variables varies considerably between patients, but 
changes in process variables and fatigue occur mostly in the same period, which suggests a complex feedback process in which fatigue and process variables influence each other.

\section{Acknowledgements}

We would like to thank all participating patients and therapists for their effort and commitment. Special thanks go to Lianne Vermeeren, Judith de Natris, Tiny Fasotti and Carel Kruip for the arduous job of data collection and Rogier Donders for his help with data imputation.

\section{Funding}

Gijs Bleijenberg received funding for this study from the Netherlands organisation for health research and development (ZON-MW). ZON-MW had no role in study design; in the collection, analysis and interpretation of data; in the writing of the report; and in the decision to submit the article for publication.

\section{Competing Interests}

The authors have no competing interests to report 


\section{References}

1. Fukuda K, Straus SE, Hickie I, Sharpe MC, Dobbins JG, Komaroff A. The chronic fatigue syndrome: a comprehensive approach to its definition and study. International Chronic Fatigue Syndrome Study Group. Annals of internal medicine. 1994;121(12):953-9.

2. Reeves WC, Lloyd A, Vernon SD, Klimas N, Jason LA, Bleijenberg G, et al. Identification of ambiguities in the 1994 chronic fatigue syndrome research case definition and recommendations for resolution. BMC health services research. 2003;3(1):25.

3. Fry AM, Martin M. Fatigue in the chronic fatigue syndrome: a cognitive phenomenon? Journal of psychosomatic research. 1996;41(5):415-26.

4. Surawy C, Hackmann A, Hawton K, Sharpe M. Chronic fatigue syndrome: a cognitive approach. Behaviour research and therapy. 1995;33(5):535-44.

5. Wessely S, David A, Butler S, Chalder T. Management of chronic (post-viral) fatigue syndrome. The Journal of the Royal College of General Practitioners. 1989;39(318):26-9.

6. Gaab J. Psychotherapie chronischer Erschöpfungszustände. Psychotherapeut. 2004;49(6):431-45.

7. Vercoulen JH, Swanink CM, Galama JM, Fennis JF, Jongen PJ, Hommes OR, et al. The persistence of fatigue in chronic fatigue syndrome and multiple sclerosis: development of a model. Journal of psychosomatic research. 1998;45(6):507-17.

8. Bleijenberg G, Prins JB, Bazelmans E. Cognitive Behavioural Therapies. In: Jason LA, Fennell PA, Taylor RR, editors. Handbook of Chronic Fatigue Syndrome. New york: Wiley \& Sons; 2003.

9. Deale A, Chalder T, Wessely S. Illness beliefs and treatment outcome in chronic fatigue syndrome. Journal of psychosomatic research. 1998;45(1 Spec No):77-83.

10. Quarmby L, Rimes KA, Deale A, Wessely S, Chalder T. Cognitive-behaviour therapy for chronic fatigue syndrome: comparison of outcomes within and outside the confines of a randomised controlled trial. Behaviour research and therapy. 2007;45(6):1085-94.

11. Sharpe M, Hawton K, Simkin S, Surawy C, Hackmann A, Klimes I, et al. Cognitive behaviour therapy for the chronic fatigue syndrome: a randomized controlled trial. BMJ (Clinical research ed. 1996;312(7022):22-6.

12. Malouff JM, Thorsteinsson EB, Rooke SE, Bhullar N, Schutte NS. Efficacy of cognitive behavioral therapy for chronic fatigue syndrome: a meta-analysis. Clinical psychology review. 2008;28(5):736-45.

13. Price JR, Mitchell E, Tidy E, Hunot V. Cognitive behaviour therapy for chronic fatigue syndrome in adults. Cochrane database of systematic reviews (Online). 2008(3):CD001027.

14. Wiborg JF, Knoop H, Frank LE, Bleijenberg G. Towards an evidence-based treatment model for cognitive behavioral interventions focusing on chronic fatigue syndrome. Journal of psychosomatic research. 2012;72(5):399-404. Epub 2012/04/04.

15. Wiborg JF, Knoop H, Stulemeijer M, Prins JB, Bleijenberg G. How does cognitive behaviour therapy reduce fatigue in patients with chronic fatigue syndrome? The role of physical activity. Psychological medicine. 2010;40(8):1281-7.

16. Puetz TW, Flowers SS, O'Connor PJ. A randomized controlled trial of the effect of aerobic exercise training on feelings of energy and fatigue in sedentary young adults with persistent fatigue. Psychotherapy and psychosomatics. 2008;77(3):167-74. Epub 2008/02/16.

17. Moss-Morris R, Sharon C, Tobin R, Baldi JC. A randomized controlled graded exercise trial for chronic fatigue syndrome: outcomes and mechanisms of change. Journal of health psychology. 2005;10(2):245-59.

18. Knoop H, van der Meer JW, Bleijenberg G. Guided self-instructions for people with chronic fatigue syndrome: randomised controlled trial. Br J Psychiatry. 2008;193(4):340-1.

19. Tummers $M$, Knoop $H$, Bleijenberg G. Effectiveness of stepped care for chronic fatigue syndrome: a randomized noninferiority trial. Journal of consulting and clinical psychology. 2010;78(5):724-31. Epub 2010/09/30. 
20. Wiborg JF, Knoop H, Prins JB, Bleijenberg G. Does a decrease in avoidance behavior and focusing on fatigue mediate the effect of cognitive behavior therapy for chronic fatigue syndrome? Journal of psychosomatic research. 2011;70(4):306-10.

21. Vercoulen JH, Bazelmans E, Swanink CM, Fennis JF, Galama JM, Jongen PJ, et al. Physical activity in chronic fatigue syndrome: assessment and its role in fatigue. Journal of psychiatric research. 1997;31(6):661-73.

22. Bergner M, Bobbitt RA, Pollard WE, Martin DP, Gilson BS. The sickness impact profile: validation of a health status measure. Medical care. 1976;14(1):57-67.

23. Jacobs HM, Luttik A, Touw-Otten FW, de Melker RA. De 'sickness impact profile'; resultaten van een valideringsonderzoek van de Nederlandse versie. [The sickness impact profile; results of an evaluation study of the Dutch version]. Nederlands tijdschrift voor geneeskunde. 1990;134(40):19504.

24. Pollard WE, Bobbitt RA, Bergner M, Martin DP, Gilson BS. The Sickness Impact Profile: reliability of a health status measure. Medical care. 1976;14(2):146-55.

25. Knoop H, Bleijenberg G, Gielissen MF, van der Meer JW, White PD. Is a full recovery possible after cognitive behavioural therapy for chronic fatigue syndrome? Psychotherapy and psychosomatics. 2007;76(3):171-6.

26. Prins JB, Bazelmans E, Van der Werf SP, Van der Meer JW, Bleijenberg G. Cognitive behaviour therapy for chronic fatigue syndrome: predictors of treatment outcome. In: Sivik T, Byrne D, Lpisitt DR, Christodoulou GN, editors. Psycho-neuro-immunology a common language for the whole body. Amsterdam: Elsevier; 2002. p. 131-5.

27. van der Werf SP, Prins JB, Vercoulen JH, van der Meer JW, Bleijenberg G. Identifying physical activity patterns in chronic fatigue syndrome using actigraphic assessment. Journal of psychosomatic research. 2000;49(5):373-9.

28. Knoop H, Bleijenberg G. Het chronisch vermoeidheidssyndroom: behandelprotocol cognitieve gedragstherapie voor CVS. Houten: Bohn, Stafleu en van Loghum; 2010.

29. Vercoulen JH, Swanink CM, Fennis JF, Galama JM, van der Meer JW, Bleijenberg G. Dimensional assessment of chronic fatigue syndrome. Journal of psychosomatic research. 1994;38(5):383-92.

30. De Vree B, Van der Werf S, Prins JB, Bazelmans E, vercoulen JH, Servaes $P$, et al. Meetinstrumenten bij chronische vermoeidheid. [Measurement instruments in chronic fatigue]. Gedragstherapie. 2002;35:157-64.

31. Prins JB, Bleijenberg G, Bazelmans E, Elving LD, de Boo TM, Severens JL, et al. Cognitive behaviour therapy for chronic fatigue syndrome: a multicentre randomised controlled trial. Lancet. 2001;357(9259):841-7.

32. Ray C, Weir W, Stewart D, Miller P, Hyde G. Ways of coping with chronic fatigue syndrome: development of an illness management questionnaire. Social science \& medicine (1982). 1993;37(3):385-91.

33. Steward AL HR, Ware JE Jr. The MOS short form general health survey: reliability and validity in a patient population. Medical care. 1998;26:724-35.

34. van der Zee K, Sanderman R. [Measuring overall health Status with the MOS SF36: a manual]. Groningen: Noordelijk Centrum voor Gezondheidsvraagstukken.

35. Scheeres $\mathrm{K}$, Wensing M, Knoop H, Bleijenberg G. Implementing cognitive behavioral therapy for chronic fatigue syndrome in a mental health center: a benchmarking evaluation. Journal of consulting and clinical psychology. 2008;76(1):163-71.

36. Bentler PM. Comparative fit indexes in structural models. Psychological bulletin. 1990;107(2):238-46.

37. Browne MW, Cudeck R. Alternative ways of assessing model fit. In: Bollen KA, Long JS, editors. Testing structural equation models. Newburt Park: Sage publications; 1993.

38. Kline RB. Principles and practice of structural equation modeling (2nd ed.): New York, NY, US: Guilford Press; 2005. 
39. Bollen KA. Overall fit in covariance structure models. Two types of sample size effects. Psychological bulletin. 1990;107:256-9.

40. Cohen J. Statistical power analysis for the behavioural sciences. 2nd ed. Hillsdale, NJ: Erlbaum; 1988.

41. Anderson EM, Lambert MJ. A survival analysis of clinically significant change in outpatient psychotherapy. J Clin Psychol. 2001;57(7):875-88. Epub 2001/06/15.

42. Wiborg JF, Knoop H, Wensing M, Bleijenberg G. Therapist effects and the dissemination of cognitive behavior therapy for chronic fatigue syndrome in community-based mental health care. Behaviour research and therapy. 2012;50(6):393-6. Epub 2012/04/17.

43. Deary V, Chalder T. Personality and perfectionism in chronic fatigue syndrome: A closer look. Psychology \& Health. 2008;25(4):465-75.

44. Gielissen MF, Knoop H, Servaes P, Kalkman JS, Huibers MJ, Verhagen S, et al. Differences in the experience of fatigue in patients and healthy controls: patients' descriptions. Health and quality of life outcomes. 2007;5:36.

45. Knoop H, van Kessel K, Moss-Morris R. Which cognitions and behaviours mediate the positive effect of cognitive behavioural therapy on fatigue in patients with multiple sclerosis? Psychological medicine. 2010:1-9.

46. Hamagami F, McArdle JJ. Dynamic extensions in Latent Change Score Models. In: Boker SM, Wenger MJ, editors. Data analytic techniques for dynamical systems (Notre Dame series on Quantitative Methodology). Mahwah (NJ), U.S.: Lawrence Erlbaum Associates Publishers; 2007. p. 47-85. 
Table 1: Structural Equation Model of change in process variables predicting change in fatigue

\begin{tabular}{|c|c|c|c|c|c|}
\hline Dependent & Predictor & Estimate & S.E. & Est./S.E. & Two-sided P-Value \\
\hline \multirow[t]{5}{*}{$\Delta$ Fatigue 1} & $\Delta$ Sense of control 1 & -0.221 & 0.059 & -3.734 & $<0.001$ \\
\hline & $\Delta$ Focus 1 & 0.195 & 0.059 & 3.297 & 0.001 \\
\hline & $\Delta$ Perc Act 1 & 0.333 & 0.061 & 5.453 & $<0.001$ \\
\hline & $\Delta$ Actual Act 1 & -0.056 & 0.060 & -0.927 & 0.35 \\
\hline & $\Delta$ Phys Funct 1 & -0.204 & 0.058 & -3.510 & $<0.001$ \\
\hline \multirow[t]{10}{*}{$\Delta$ Fatigue 2} & $\Delta$ Sense of control 1 & -0.091 & 0.062 & -1.471 & 0.14 \\
\hline & $\Delta$ Focus 1 & -0.095 & 0.065 & -1.455 & 0.15 \\
\hline & $\Delta$ Perc Act 1 & -0.027 & 0.068 & -0.396 & 0.69 \\
\hline & $\Delta$ Actual Act 1 & 0.053 & 0.066 & 0.808 & 0.42 \\
\hline & $\Delta$ Phys Funct 1 & -0.186 & 0.061 & -3.026 & 0.002 \\
\hline & $\Delta$ Sense of control 2 & -0.263 & 0.061 & -4.341 & $<0.001$ \\
\hline & $\Delta$ Focus 2 & 0.147 & 0.059 & 2.503 & 0.01 \\
\hline & $\Delta$ Perc Act 2 & 0.367 & 0.056 & 6.507 & $<0.001$ \\
\hline & $\Delta$ Actual Act 2 & 0.020 & 0.062 & 0.315 & 0.75 \\
\hline & $\Delta$ Phys Funct 2 & -0.242 & 0.058 & -4.154 & $<0.001$ \\
\hline \multirow[t]{9}{*}{$\Delta$ Fatigue 3} & $\Delta$ Sense of control 1 & 0.039 & 0.051 & 0.763 & 0.45 \\
\hline & $\Delta$ Focus 1 & 0.049 & 0.054 & 0.899 & 0.37 \\
\hline & $\Delta$ Perc Act 1 & -0.030 & 0.059 & -0.517 & 0.61 \\
\hline & $\Delta$ Actual Act1 & 0.006 & 0.065 & 0.008 & 0.93 \\
\hline & $\Delta$ Phys Funct 1 & 0.026 & 0.053 & 0.494 & 0.62 \\
\hline & $\Delta$ Sense of control 2 & -0.013 & 0.058 & -0.221 & 0.83 \\
\hline & $\Delta$ Focus 2 & -0.003 & 0.056 & -0.059 & 0.95 \\
\hline & $\Delta$ Perc Act 2 & 0.024 & 0.059 & 0.412 & 0.68 \\
\hline & $\Delta$ Actual Act 2 & -0.001 & 0.078 & -0.018 & 0.99 \\
\hline
\end{tabular}




\begin{tabular}{|c|c|c|c|c|c|}
\hline & $\Delta$ Phys Funct 2 & -0.030 & 0.057 & -0.525 & 0.60 \\
\hline & $\Delta$ Sense of control 3 & -0.249 & 0.049 & -5.074 & $<0.001$ \\
\hline & $\Delta$ Focus 3 & 0.151 & 0.053 & 2.854 & 0.004 \\
\hline & $\Delta$ Perc Act 3 & 0.391 & 0.053 & 7.439 & $<0.001$ \\
\hline & $\Delta$ Actual Act 3 & -0.017 & 0.064 & -0.271 & 0.79 \\
\hline & $\Delta$ Phys Funct 3 & -0.353 & 0.049 & -7.160 & $<0.001$ \\
\hline$\Delta$ Fatigue 2 & $\Delta$ Fatigue 1 & -0.367 & 0.065 & -5.627 & $<0.001$ \\
\hline \multirow[t]{2}{*}{$\Delta$ Fatigue 3} & $\Delta$ Fatigue 1 & -0.083 & 0.077 & -1.075 & 0.28 \\
\hline & $\Delta$ Fatigue 2 & -0.359 & 0.066 & -5.464 & $<0.001$ \\
\hline \multirow[t]{3}{*}{ Intercepts } & $\Delta$ Fatigue 1 & -0.288 & 0.091 & -3.150 & 0.002 \\
\hline & $\Delta$ Fatigue 2 & -0.090 & 0.091 & -0.988 & 0.32 \\
\hline & $\Delta$ Fatigue 3 & -0.185 & 0.084 & -2.204 & 0.03 \\
\hline
\end{tabular}


Footnote table 1: Estimates are the predicted change in fatigue when the predictor increases 1 point; $1=$ interim measurement $1-$ pre measurement; $2=$ interim measurement $2-1 ; 3=$ post measurement - interim measurement 2; Fatigue $=$ Checklist Individual Strength subscale fatigue severity; Sense of control= Self-Efficacy Scale; Focus= subscale focusing Illness Management Questionnaire; Perc Act=subscale perceived activity of the Checklist Individual Strength; Obj act=mean daily activity score as measured with the actometer; $\mathrm{X}^{2}=20.75(\mathrm{df}=12) \mathrm{p}=.054 ; \mathrm{CFI}=.97$; RMSEA $=0.07(90 \% \mathrm{Cl} 0.00-0.13) ; r^{2}$ period $1=.50$, period $2=.55$, period $3=.63$ 
Figure 1: Potential process variables that we tested, based on the models of Wiborg (14) and Vercoulen (29).

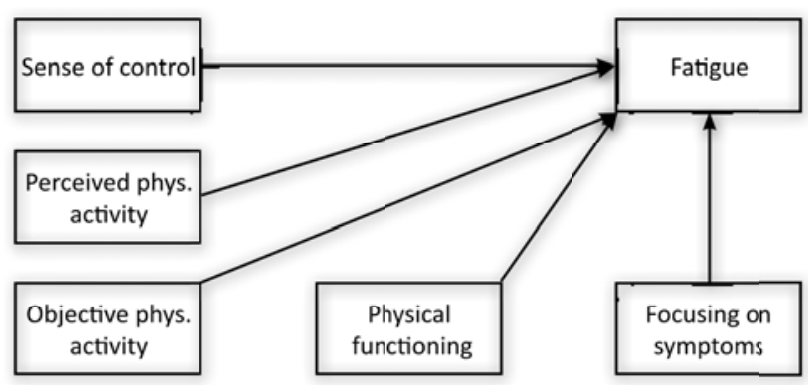


Figure 2: Graphical representation of the structural equation model

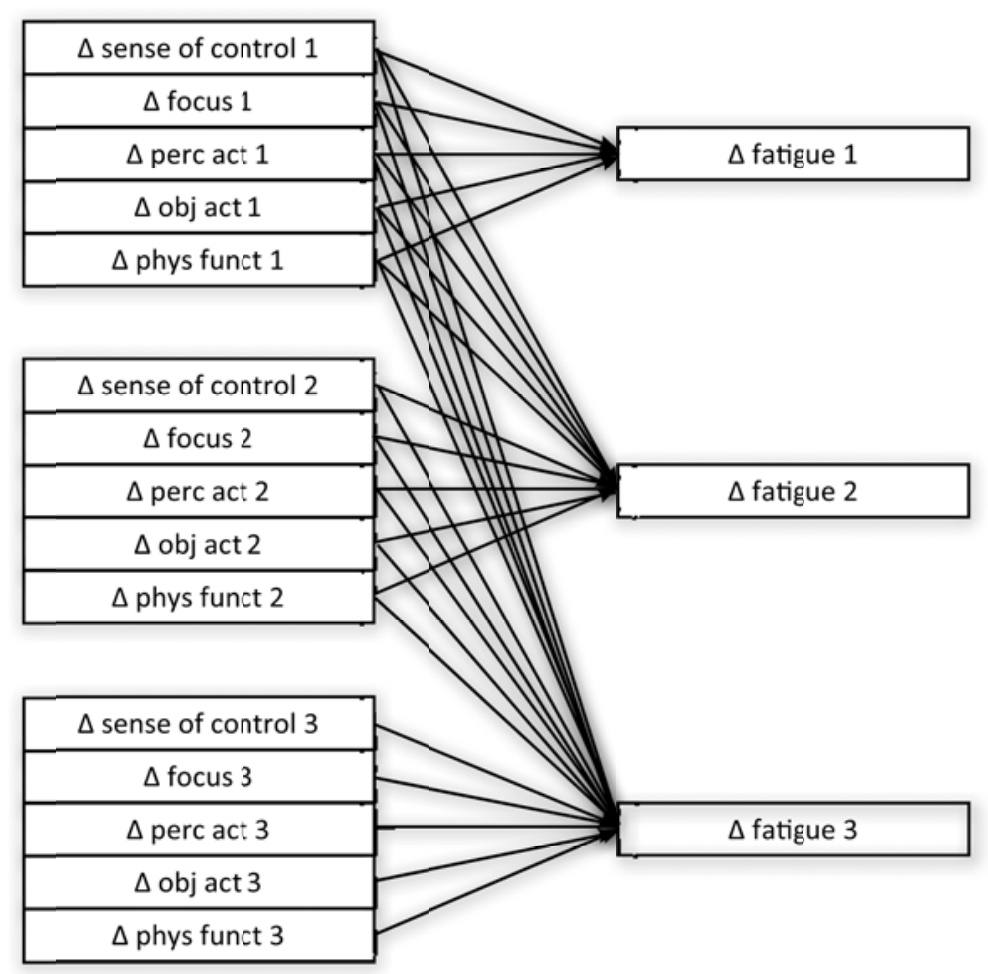

Footnote: Fatigue=Checklist Individual Strength subscale fatigue severity; Perceived activity=Checklist Individual Strength subscale physical activity; Actual activity=mean daily score as measured with actometer; sense of control=SES28 total score; focusing on symptoms=subscale focusing on symptoms of the Illness Management Questionnaire 
Figure 3: Flow chart of the selection of the study participants

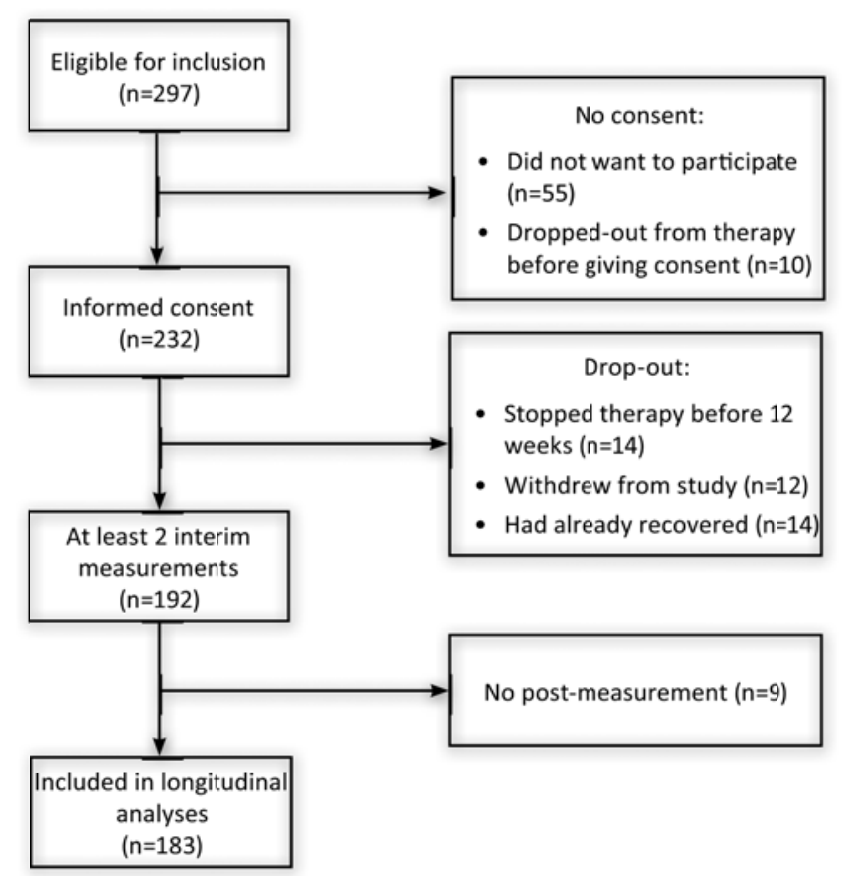


Figure 4

Caption: Mean fatigue and process variables during therapy in the different response groups

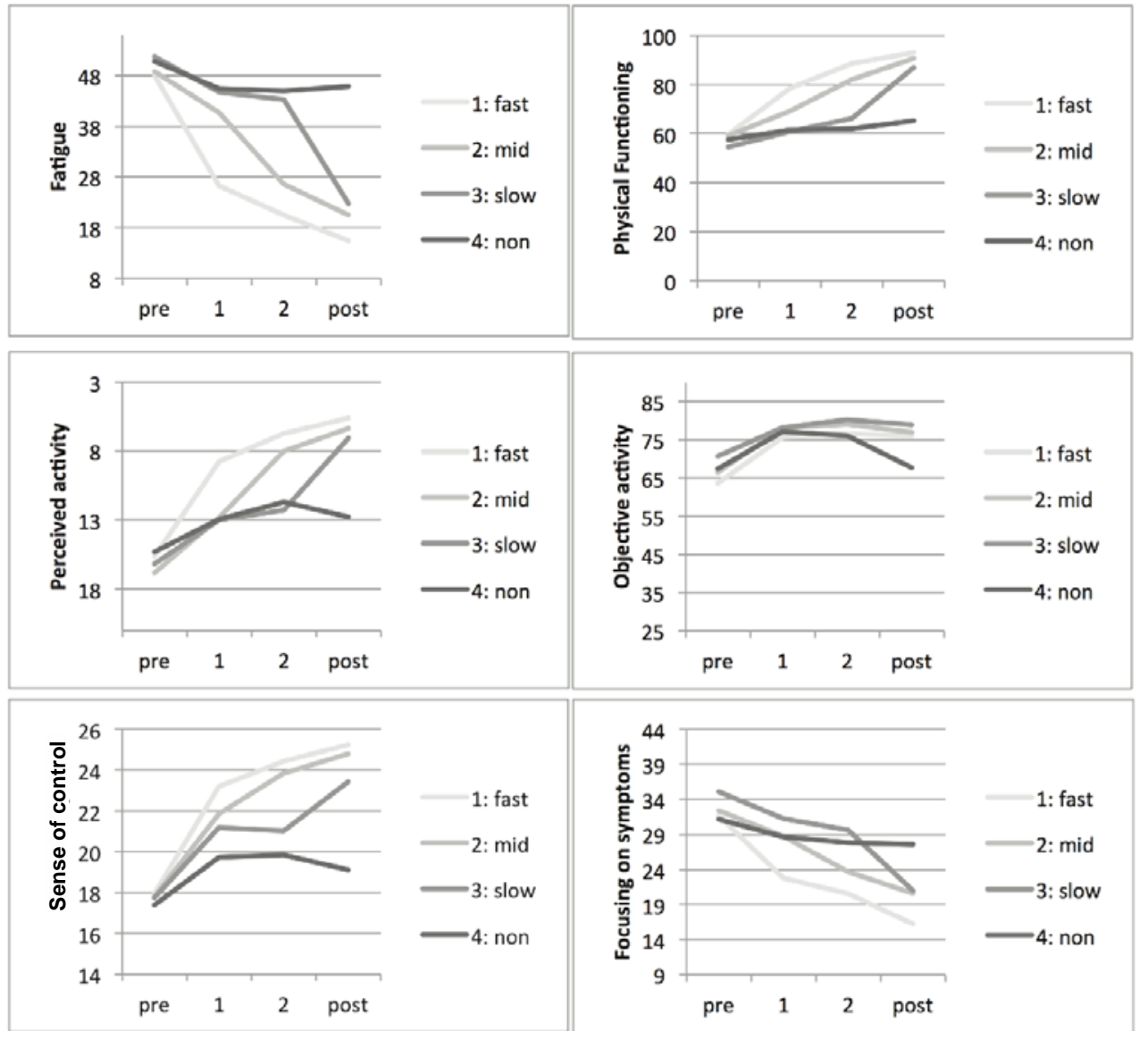

Footnote: Fast=fast responders; mid=mid-term responders; slow=slow responders; non=non responders; Fatigue=Checklist Individual Strength subscale fatigue severity; Perceived activity=Checklist Individual Strength subscale physical activity; activity=mean daily score as measured with actometer; sense of control=SES28 total score; focusing on symptoms=subscale focusing on symptoms of the Illness Management Questionnaire 